Applied and

NISTIR 4662

Computational

Mathematics

Division

Computing and Applied Mathematics Laboratory

\title{
A Phase-Field Model For Isothermal Phase Transitions In Binary Alloys
}

A. A. Wheeler, W. J. Boettinger, and G. B. McFadden August 1991

U.S. DEPARTMENT OF COMMERCE

$-Q C$ 100 .056 4662 1991

C. 2 



\section{A Phase-Field Model For Isothermal Phase Transitions In Binary Alloys}
A. A. Wheeler,
W. J. Boettinger,
G. B. McFadden

U.S. DEPARTMENT OF COMMERCE National Institute of Standards and Technology Applied and Computational Mathematics Division

Gaithersburg, MD. 20899

August 1991

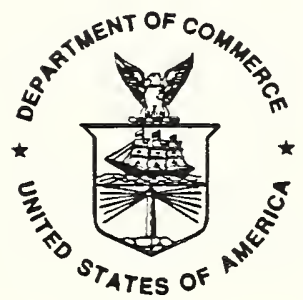

U.S. DEPARTMENT OF COMMERCE Robert A. Mosbacher, Secrotary MATIONAL INSTIUUTE OF STANDARDS AND TECHNOLOGY

John W. Lyons, Director 



\title{
A Phase-Field Model For Isothermal Phase Transitions In Binary Alloys
}

\author{
A. A. Wheeler;, W. J. Boettinger, and G. B. McFadden \\ National Institute of Standards and Technology \\ Gaithersburg, MD 20899 \\ USA
}

\begin{abstract}
In this paper we describe a new phase-field model to describe isothermal phase transitions between ideal binary alloy solution phases. Governing equations are developed for the temporal and spatial variation of the phase field, which identifies the local state or phase, and for the composition. An asymptotic analysis as the gradient energy coefficient of the phase-field becomes small shows that our model recovers classical sharp interface models of this situation when the interfacial layers are thin, and we relate the parameters appearing in the phase-field model to material and growth parameters in real systems. We identify three stages of temporal evolution for the governing equations; the first corresponds to interfacial genesis which occurs very rapidly; the second to interfacial motion controlled by diffusion and the local energy difference across the interface; the last takes place on a long time scale in which curvature effects are important, and corresponds to Ostwald ripening. We also present results of numerical calculations.
\end{abstract}

*Permanent address: School Of Mathematics, University Walk, Bristol, BS8 1TW, UK. 


\section{Introduction}

Classical macroscopic models of phase transitions describe the interface between regions of different phase as a surface of zero thickness. The governing equations for thermodynamic variables, such as temperature and composition, are formulated in each phase independently, based on conservation principles and quantitatively verified phenomenological laws that relate fluxes to gradients. The boundary conditions at the interface are of two types: those which represent conservation laws when there are discontinuities in the thermodynamic variables or their gradients, and those which deal with the values of the thermodynamic variables at the interface and are based on modeling of the interface on an atomic scale. This approach gives rise to the formulation of a free boundary problem which provides a difficult mathematical setting in which only phase changes with simple configurations have been rigorously analysed mathematically. Because these models have been used for many years, it is clear from the outset what physical mechanisms are incorporated into them, and comparison with carefully controlled experiments have been performed, e.g. the dendritic growth experiments of Huang and Glicksman [1] and the grain boundary groove measurements of Hardy [2].

An alternative technique for investigating systems involving a phase transition involves the construction of a Cahn-Hilliard or Landau-Ginzberg free energy functional, which unlike the classical model treats the system as a whole. This approach has its roots in statistical physics (see e.g. Landau and Khalatinikov [3]). For this purpose, a phase field, $\phi(x, t)$, is postulated to exist, which characterises the phase of the system at each point in space and time. It is assumed that the Helmholtz free energy $\mathcal{F}[\phi, \ldots]$, is a functional of the phase field, as well as any other thermodynamic variables (such as temperature and composition which are denoted here by ellipsis), and their gradients, in the following way:

$$
\mathcal{F}[\phi, \ldots]=\int_{\Omega}\left[f(\phi, \ldots)+\frac{1}{2} \epsilon^{2}(\nabla \phi)^{2}+\ldots\right] d \Omega,
$$

where $\Omega$ is the region occupied by the system, and $f(\phi, \ldots)$ is the Helmholtz free energy density for a phase with no gradients. Its dependence on $\phi$ usually has a "double-well" form. Here we assume that the only contribution to the energy functional from local gradients is that of the phase field; a more general model might also include contributions due to the gradients of the other thermodynamic variables. The phase field is then assumed to evolve 
as:

$$
\dot{\phi} \propto \mathcal{L}\left(\frac{\delta \mathcal{F}}{\delta \phi}\right),
$$

where $\mathcal{L}$ is some partial differential operator, such that $\mathcal{L}(0)=0$. This equation is then supplemented by partial differential equations for the other thermodynamic variables.

In some situations the composition naturally plays the role of the phase field, in which case the double-well in the free energy occurs with respect to composition. Cahn and Hilliard $[4,5,6]$ have used this approach to model interfacial energies, nucleation and spinodal decomposition in a binary alloy. Also Langer and Sekerka [7] have modeled the motion of a planar interface using this approach.

More generally, various models that employ these ideas are reviewed by Halperin, Hohenburg and $\mathrm{Ma}$ [8], particularly in regard to the study of critical phenomena. The Model $\mathrm{C}$ given by Halperin et al. has been adapted by Langer [9], Fix [10], and most prolifically by Caginalp [11], to derive the so-called "phase-field model" of solidification which describes the phase change of a pure material. Caginalp has studied this model, and its variations $[12,13]$, extensively. In this model the phase field is required to evolve according to:

$$
\alpha \xi^{2} \phi_{t}=-\frac{\delta \mathcal{F}}{\delta \phi}=\xi^{2} \nabla^{2} \phi+\frac{1}{2 a} \phi\left(1-\phi^{2}\right)+2 T,
$$

where $a, \alpha$, and $\xi$ are non-dimensional constants. A non-dimensional temperature, $T$, satisfies the heat equation, modified to allow for latent heat production as a source term proportional to $\phi_{t}$ :

$$
T_{t}+\frac{l}{2} \phi_{t}=K \nabla^{2} T
$$

where $l$ is the latent heat production. The Helmholtz free energy density used by Caginalp is:

$$
f(\phi, T)=\frac{1}{8 a}\left(\phi^{2}-1\right)^{2}-2 T \phi,
$$

where $T=0$ is the phase transition temperature.

It has emerged from study of this model that qualitatively it exhibits features common to solidification of a pure material. Numerical calculations by Smith [14] and by Socolowski and Caginalp [15], and for a similar model by Kobayashi [16], show such features as breakdown of planar and circular interfaces to cellular structures, as well as the formation of dendrite-like structures, inclusion of liquid pockets, and Ostwald ripening or coarsening behaviour. 
Caginalp [12] has shown in various distinguished limits, in which $\xi \rightarrow 0$, that various forms of the classical Stefan problem may be recovered, in which the interface is taken to be "sharp" i.e. modeled by a surface. In this limit, there are thin layers within $\Omega$ of thickness $\mathcal{O}(\xi)$ in which the phase field rapidly changes. These are interpreted as representing diffuse interfaces. From this analysis it transpires that in some limits, the interfacial dynamics involve curvature effects corresponding to the Gibbs-Thomson interfacial surface energy effect as well as kinetic effects. In other limits, it is also possible to recover the classical Hele-Shaw problem. It is clear that this approach can embody a considerable variety of realistic physical effects in a coherent way.

When applying the model above to a definite physical situation it is not clear how to choose the values of parameters in the phase-field model so that it describes the solidification of a pure material with known materials and growth parameters. Such a choice of parameters is essential to permit comparison with experiment. Also the free energy functional is only employed in the formulation of the kinetic equation for the phase field and not for the temperature. An alternative approach, as suggested by Penrose and Fife [17], is to construct an entropy functional of the system, and postulate kinetic equations for $\phi$ and $T$ which ensure that the entropy increases monotonically in time, as required by the Second Law of Thermodynamics.

The appeal of phase-field models in describing phase transitions is twofold:

- It provides a simple, elegant description, that appears to embody a rich variety of realistic physical growth phenomena.

- From a computational point of view, it is relatively simple to obtain solutions. This is because it is not necessary to distinguish between the different phases. Computations that employ the classical sharp interface formulation require the free boundary to be tracked numerically, and the regions occupied by each phase are therefore determined and dealt with individually. Added difficulties with the classical method are encountered when the connectedness of the solid or liquid regions change in time. These considerations often result in very difficult and untidy numerical algorithms.

In this paper we derive and analyse a new phase-field model for isothermal phase tran- 
sitions of a solution of two components. In an isothermal system the driving force for phase transformation is developed through the growth of a product phase into a supersaturated parent phase. To our knowledge, there are to date no phase-field models that deal with impure materials and alloys. The model presented here is a first step to developing a phase-field model for the solidification of an alloy. In future work we hope to incorporate this model in a formulation based on increasing entropy, which will then allow both variations in temperature and composition, and provide a phase-field model for commercially important alloy solidification processes used in such things as the fabrication of modern electronic devices and directionally solidified turbine alloys.

In section 2 we discuss the phase-field model of a pure material due to Kobayashi [16], and use it as a basis to derive our isothermal phase-field model for growth of binary alloys. We employ a kinetic formulation which ensures that the Helmholtz free energy decreases monotonically in time. In the following section we consider the sharp interface limit. This shows the correspondence of our phase-field model with classical sharp interface models, and allows us to relate the materials parameters in the phase-field model to those of the classical Stefan problem.

We show that the time evolution of the system occurs on three scales. There is an initial very short period in which interfaces form. It is a response of the system to the initial data and does not necessarily represent the process of nucleation. This is followed by a second period in which the interfaces move in response to the energy density difference across them. In the third and final period coarsening occurs, in which the interfaces move very slowly, primarily in response to changes in curvature. In section 4 we discuss the motion of a planar interface and show how segregation is dependent on the interfacial growth rate in this model. In the last section, we present results from a numerical integration of the model, which bear out the results of section 3 and 4 and show the power of this formulation. 


\section{Problem Formulation}

\subsection{The Governing Equations}

We consider an isothermal solution of two different components $A$ and $B$ which may exist as two distinct phases, solid and liquid, contained in a fixed region $\Omega$ with boundary $\partial \Omega$. We denote the concentration of $\mathrm{B}$ by $c(\mathbf{x}, t)$ and we introduce a phase field $\phi(\mathbf{x}, t)$ which represents the phase in time and space in $\Omega$. Specifically, we describe the solid-liquid interface by $\phi(\mathbf{x}, t)=1 / 2$ and denote regions where $\phi(\mathbf{x}, t)>1 / 2$ as solid and regions where $\phi(x, t)<$ $1 / 2$ as liquid.

A recent phase-field model due to Kobayashi [16] models the phase transition of a pure material by employing the following gradient weighted free-energy functional:

$$
\mathcal{F}[\phi, T]=\int_{\Omega}\left[f(\phi, T)+\frac{\epsilon^{2}}{2}|\nabla \phi|^{2} f(\phi, T)\right] d \Omega,
$$

where $\epsilon$ is a constant, $T(\mathbf{x}, t)$, is the temperature and the free energy density is

$$
f(\phi, T)=\int_{0}^{\phi} p(p-1)\left(p-\frac{1}{2}-\beta(T)\right) d p .
$$

Here $\beta(T)$ is a monotonic increasing function of $T$, such that $\beta\left(T_{M}\right)=0$, where $T_{M}$ is the freezing temperature of the material, and $|\beta(T)|<1 / 2$. The free energy density $f(\phi, T)$ is a double-well potential in $\phi$, see Figure 1 . The restriction $|\beta(T)|<1 / 2$ ensures that it has local minima at $\phi=0$ and $\phi=1$, and a local maximum at $\phi=1 / 2+\beta(T)$. Because of the two minima, the system may exist stably in a state which is a single phase liquid $(\phi(\mathbf{x}, t) \equiv 0)$ or a single phase solid $(\phi(\mathbf{x}, t) \equiv 1)$. There is an energy penalty for a change of phase within the region $\Omega$, which corresponds to $\phi$ varying between zero and unity. This is because such a variation increases the total energy $\mathcal{F}$ of the system, due to an increased energy density associated with the double-well nature of the energy density, and also due to the contribution to the total energy due to the gradient energy, which is no longer zero.

If $-1 / 2<\beta<0$, i.e $T<T_{M}$, then the global minimum of the energy density is at $\phi=1$, and so the single-phase solid has the lowest energy. However, if $0<\beta<1 / 2$, i.e $T>T_{M}$ then the situation is reversed, and the single-phase liquid has the lowest energy.

We now employ this form for the free energy density to develop the appropriate free energy density for an isothermal solution in which the two different phases are assumed to be 
ideal solutions. We assume that $T_{M}^{A}>T_{M}^{B}$, where $T_{M}^{A}$ and $T_{M}^{B}$ are the freezing temperatures of pure $A$ and pure $B$ when $c=0$ and $c=1$ respectively. We assume that the actual temperature of the solution, $T$, which is given, lies between these two freezing temperatures, i.e. $T_{M}^{A}>T>T_{M}^{B}$. We also assume that the Helmholtz free energy densities of each component $\mathrm{A}$ and $\mathrm{B}$ alone are of the form given by Kobayashi, and are denoted by $f_{A}(\phi ; T)$ and $f_{B}(\phi ; T)$ respectively. Specifically we put:

$$
\begin{aligned}
& f_{A}(\phi ; T)=W_{A} \int_{0}^{\phi} p(p-1)\left(p-\frac{1}{2}-\beta_{A}(T)\right) d p \\
& f_{B}(\phi ; T)=W_{B} \int_{0}^{\phi} p(p-1)\left(p-\frac{1}{2}-\beta_{B}(T)\right) d p
\end{aligned}
$$

where $W_{A}, W_{B}$ are constants, and the temperature $T$ is a parameter in this isothermal situation. We note that because $T_{M}^{B}<T<T_{M}^{A}$, then $-1 / 2<\beta_{A}(T)<0<\beta_{B}(T)<1 / 2$. We take the energy density $f(\phi, c ; T)$ of the solution as:

$$
f(\phi, c ; T)=c f_{B}(\phi ; T)+(1-c) f_{A}(\phi ; T)+\frac{R T}{v_{m}}[c \log c+(1-c) \log (1-c)],
$$

where $R$ is the universal gas constant constant and $v_{m}$ is the molar volume, which is assumed to be constant. The first two terms correspond to the contribution to the energy density due to the individual molar Helmholtz free energies densities of the two components and the last term is due to the decrease in energy associated with the mixing of the two components, under our assumption that both liquid and solid phases are ideal solutions. In Figure 2 we represent the free energy density (10) as a surface. This model could easily be extended to the case of a regular solution by adding a term of the form $c(1-c)\left[\Omega_{L}+\phi\left(\Omega_{S}-\Omega_{L}\right)\right]$ to the free-energy density $f(c, \phi)$, where $\Omega_{L}$ and $\Omega_{S}$ are thermodynamic constants.

We define the free energy functional by:

$$
\mathcal{F}[\phi, c ; T]=\int_{\Omega}\left[f(\phi, c ; T)+\frac{\epsilon^{2}}{2}|\nabla \phi|^{2}\right] d \Omega .
$$

In order to derive a kinetic model we make the assumption that the system evolves in time so that its total free energy decreases monotonically. The following prescription is consistent with this assumption and ensures that the total amount of solute is conserved:

$$
\frac{\partial \phi}{\partial t}=-M_{1} \frac{\delta \mathcal{F}}{\delta \phi}
$$




$$
\frac{\partial c}{\partial t}=M_{2} \nabla \cdot\left(c(1-c) \nabla \frac{\delta \mathcal{F}}{\delta c}\right)
$$

where $M_{1}$ and $M_{2}$ are positive constants. The choice of boundary conditions

$$
\frac{\partial \phi}{\partial n}=\frac{\partial c}{\partial n}=0
$$

where $\mathbf{n}$ is the outward normal to the boundary $\partial \Omega$, ensures that there is no change in the total composition of the system due to transport across its boundary and that the Helmholtz free energy decreases monotonically with time. We may interpret the right hand side of (13) as the negative of the divergence of a solute flux, $\mathrm{j}=-M_{2} c(1-c) \nabla(\delta \mathcal{F} / \delta c)$. The coefficient $c(1-c)$ has been included to ensure that the diffusion equation for the solute that emerges has a diffusion coefficient that is not dependent upon composition.

Evaluating the variational derivatives of the free energy functional gives:

$$
\begin{gathered}
\frac{\partial \phi}{\partial t}=M_{1}\left(\epsilon^{2} \nabla^{2} \phi-\frac{\partial f}{\partial \phi}\right), \\
\frac{\partial c}{\partial t}=M_{2} \nabla \cdot\left(c(1-c) \nabla \frac{\partial f}{\partial c}\right),
\end{gathered}
$$

which may be also written by using (10) as:

$$
\begin{aligned}
& \frac{\partial \phi}{\partial t}=M_{1}\left[\epsilon^{2} \nabla^{2} \phi-\left(c \frac{\partial f_{A}}{\partial \phi}+(1-c) \frac{\partial f_{B}}{\partial \phi}\right)\right], \\
& \frac{\partial c}{\partial t}=M_{2} \nabla \cdot\left(c(1-c) \nabla\left(f_{A}-f_{B}\right)\right)+D \nabla^{2} c
\end{aligned}
$$

where $D=M_{2} R T / v_{m}$ is identified as the diffusivity of the solute. We note that in this formulation the solute diffusivity is the same in each phase; this restriction could be overcome by allowing the quantity $M_{2}$ to depend on $\phi$.

We now nondimensionalise these governing equations by introducing the following dimensionless quantities (denoted by a tilde):

$$
\mathbf{x}=l^{*} \tilde{\mathbf{x}}, \quad t=\frac{l^{* 2}}{D} \tilde{t}, \quad T=T^{*} \tilde{T}, \quad f=\frac{R T}{v_{m}} \tilde{f},
$$

where $l^{*}$ is a representative length of the system $\Omega, T^{*}=\left(T_{M}^{A}+T_{M}^{B}\right) / 2$ is the average of the melting temperatures of the two constituents, and time has been scaled on the diffusive time scale. Here $\tilde{f}=\tilde{f}(\phi, c ; \tilde{T})=c \tilde{f}_{B}(\phi ; \tilde{T})+(1-c) \tilde{f}_{A}(\phi ; \tilde{T})+[c \log c+(1-c) \log (1-c)]$, where 
$\tilde{f}_{A}(\phi ; \tilde{T})=\widetilde{W}_{A} \int_{0}^{\phi} p(p-1)\left(p-\frac{1}{2}-\beta_{A}(\tilde{T})\right) d p, \tilde{f}_{B}(\phi ; \tilde{T})=\widetilde{W}_{B} \int_{0}^{\phi} p(p-1)\left(p-\frac{1}{2}-\beta_{B}(\tilde{T})\right) d p$ $\widetilde{W}_{A}=W_{A} v_{m} / R T$ and $\widetilde{W}_{B}=W_{B} v_{m} / R T$.

The governing equations, (17) and (18) can then be written as

$$
\begin{aligned}
& \frac{\partial \phi}{\partial \tilde{t}}=\tilde{M}_{1}\left(\tilde{\epsilon}^{2} \tilde{\nabla}^{2} \phi-\frac{\partial \tilde{f}}{\partial \phi}\right), \\
& \frac{\partial c}{\partial \tilde{t}}=\tilde{\nabla} \cdot\left(c(1-c) \tilde{\nabla} \frac{\partial \tilde{f}}{\partial c}\right),
\end{aligned}
$$

where

$$
\tilde{M}_{1}=\frac{M_{1} l^{* 2} R T^{*}}{D v_{m}}, \quad \tilde{\epsilon}=\frac{\epsilon}{l^{*}} \sqrt{\frac{v_{m}}{R T^{*}}} .
$$

\subsection{Relationship Between Materials Parameters of the Phase- Field Model and Sharp Interface Theories}

In order to determine the relative magnitudes of the dimensionless parameters and to permit application to real materials systems, it is necessary to obtain estimates for the seven materials-related parameters of the alloy phase-field model. They are $\epsilon, W_{A}, W_{B}, \beta_{A}(T), \beta_{B}(T)$, $M_{1}$ and $M_{2}$. The parameter $M_{2}$ has already been related to the diffusion coefficient as $D=M_{2} R T / v_{m}$, through the similarity of (18) (when $\phi$ is identically zero or unity) to the linear diffusion equation.

We proceed to develop expressions that relate the parameters $\epsilon, W_{A}, \beta_{A}(T)$ and $M_{1}$ to parameters common to sharp interface theories for pure $\mathrm{A}$. These parameters are: $\sigma_{A}$, solidliquid surface energy of a stationary planar interface; $L_{A}$, the latent heat of fusion per unit volume; and $\mu_{A}$, the linear kinetic coefficient, which relates the normal interface velocity $V_{n}$ to interface temperature by

$$
V_{n}=\mu_{A}\left(T_{M}^{A}-T\right) .
$$

This data (or estimates of it) is available for most pure materials. The corresponding identification of parameters for the case of pure B is completely analogous. We consider each parameter separately in turn. 


\subsubsection{Energy Difference Between Phases}

The difference in free energy between solid and liquid phases is known as a function of temperature. This quantity, $f_{A}(1 ; T)-f_{A}(0 ; T)$, is often approximated by a linear function of temperature near the melting point as:

$$
f_{A}(1 ; T)-f_{A}(0 ; T)=\frac{L_{A}\left(T-T_{M}^{A}\right)}{T_{M}^{A}} .
$$

Evaluation of this free energy difference from the integration of (8) gives

$$
f_{A}(1 ; T)-f_{A}(0 ; T)=\frac{W_{A} \beta_{A}(T)}{6},
$$

hence

$$
\frac{W_{A} \beta_{A}(T)}{6}=\frac{L_{A}\left(T-T_{M}^{A}\right)}{T_{M}^{A}}
$$

\subsubsection{Free Surface Energy}

An expression for $\sigma_{A}$, the excess free energy of the interfacial region over the bulk phases has been derived for an interface with a nonconserved order parameter (the case employed in our phase-field model) by Allen and Cahn [19]. With change of notation this is

$$
\sigma_{A}=\epsilon \int_{0}^{1} \sqrt{f_{A}(\phi ; T)} d \phi .
$$

Evaluation of this integral (at $T=T_{M}$ ) using the form of the free energy density given by (8) yields

$$
\sigma_{A}=\frac{\epsilon \sqrt{W_{A}}}{12}
$$

\subsubsection{Interface Kinetics}

We consider the one-dimensional solution of (15) for a pure material, $c=0$ or $c=1$, (see Harowell and Oxtoby [20]) which represents the uniform motion of a planar, diffuse interface. Transforming to a frame moving with constant velocity $V_{n},(15)$ has a steady solution which satisfies

$$
\epsilon^{2} \frac{d^{2} \phi}{d x^{2}}+\frac{V_{n}}{M_{1}} \frac{d \phi}{d x}-\frac{\partial f}{\partial \phi}=0
$$


The only solution with the property that $\phi \rightarrow 1$ as $x \rightarrow-\infty$ and $\phi \rightarrow 0$ as $x \rightarrow+\infty$ is

$$
\phi(x)=\left[1+\exp \left(x \sqrt{\frac{W_{A}}{2 \epsilon^{2}}}\right)\right]^{-1}
$$

and this solution only exists when

$$
V_{n}=-M_{1} \epsilon \beta_{A}(T) \sqrt{2 W_{A}}
$$

We see from the form of the solution (30), or from Allen and Cahn [19], that the thickness of the interface $\delta_{A}$ is characterised as

$$
\delta_{A}=\epsilon \sqrt{\frac{2}{W_{A}}}
$$

Equations (26) and (31) give

$$
V_{n}=-6 M_{1} \epsilon L_{A} \sqrt{\frac{2}{W_{A}}}\left(\frac{T-T_{M}^{A}}{T_{M}^{A}}\right)
$$

from which by comparison with (23) we find

$$
\mu_{A}=\frac{6 M_{1} L_{A} \delta_{A}}{T_{M}^{A}}
$$

Equations (26), (28) and (34), derived from comparison with classical sharp interface theory, provide three relations for the four unknowns $\epsilon, W_{A}, \beta_{A}(T)$ and $M_{1}$. In addition, information is required about either the height of the maximum of the double-well potential ( $W_{A} / 64$ at $T=T_{M}^{A}$ ) or the interface thickness $\delta_{A}$ to uniquely establish values for all parameters. In some cases, such as spinodal decomposition, the height of the maximum of the double-well potential can be estimated from a regular solution model of the phase and the proximity of the critical point. In the present application to liquid-solid reactions, such an approach is not currently possible. We therefore choose to treat the interface thickness as an input parameter, in which case the values of the phase field are related to the classical data as:

$$
\begin{gathered}
\epsilon^{2}=6 \sqrt{2} \sigma_{A} \delta_{A} \\
W_{A}=12 \sqrt{2} \frac{\sigma_{A}}{\delta_{A}} \\
M_{1}=\frac{\mu_{A} T_{M}^{A}}{6 L_{A} \delta_{A}}
\end{gathered}
$$




$$
\beta_{A}(T)=\left(\frac{L_{A} \delta_{A}}{2 \sqrt{2} \sigma_{A}}\right)\left(\frac{T-T_{M}^{A}}{T_{M}^{A}}\right)
$$

by employing equations (26), (28), (32) and (34).

We emphasize that whatever choice we make for the interface thickness the resulting values of $\epsilon, W_{A}, \beta_{A}(T)$ and $M_{1}$ provide a model that has the required free energy change, surface energy and kinetics to match the classical theory.

To treat the alloy case at hand in the present paper, the last four equations would be evaluated for each pure component. However, because we have assumed that $\epsilon$ and $M_{1}$ are constant and do not depend on composition, we are restricted in the choices for some parameters. To ensure that single values of $\epsilon$ and $M_{1}$ are obtained, the following restrictions must be satisfied:

$$
\begin{gathered}
\frac{\delta_{A}}{\delta_{B}}=\frac{\sigma_{B}}{\sigma_{A}}, \\
\frac{\mu_{A}}{\mu_{B}}=\frac{\delta_{A}}{\delta_{B}} \frac{L_{A}}{L_{B}} \frac{T_{M}^{B}}{T_{M}^{A}} .
\end{gathered}
$$

These restrictions could be removed by choosing both $\epsilon$ and $M_{1}$ to depend linearly on composition.

Numerical values of these constants can be estimated for the alloy system $\mathrm{Ni}-\mathrm{Cu}$. The liquid-solid part of this phase diagram closely resembles that for the ideal solution model assumed in this work. Values for the parameters are taken primarily from Coriell and Turnbull [22] and are given in Table 1. The dimensionless parameters $\tilde{\epsilon}$ and $\tilde{M}_{1}$ can be calculated from the data in Table 1 . For a length scale $l^{*}$, we chose a dimension typical of cellular and dendritic structures of $100 \mu \mathrm{m}$. Using the data in Table 1 and $T^{*}=\left(T_{M}^{A}+T_{M}^{B}\right) / 2$, we have $\tilde{\epsilon}=9.6 \times 10^{-6}$, and $\tilde{M}_{1}=8.5 \times 10^{12}$. These values, along with equations (20) and (21), provide a complete formulation for the calculation of isothermal phase transition (solidification) in the $\mathrm{Ni}-\mathrm{Cu}$ alloy.

\section{Asymptotic Analysis: The Limit $\tilde{\epsilon} \rightarrow 0$.}

In this section we analyse the system in the limit $\tilde{\epsilon} \rightarrow 0$. We restrict our attention to the situation when the domain $\Omega$ is two-dimensional. We assume that $\phi$ and $c$ satisfy Neumann conditions on the boundary, so that the total amount of solute is conserved and the free 
energy of the system decreases monotonically in time. In particular, we consider the spatiotemporal evolution of the concentration and phase field from given initial data. We require that the initial data, $c_{i n i t}(\mathbf{x})$ and $\phi_{i n i t}(\mathbf{x})$ for $c(\mathbf{x}, t)$ and $\phi(\mathbf{x}, t)$ respectively is bounded in the interval $[0,1]$, i.e. $0 \leq c_{\text {init }}(\mathbf{x}) \leq 1,0 \leq \phi_{\text {init }}(\mathbf{x}) \leq 1$, and satisfies the Neumann boundary conditions. We emphasize that because of the generality of this initial data it does not necessarily represent a physically realistic initial state.

We assume that the contribution of the gradient energy is much smaller than that of the free energy density and so $\epsilon^{2} / l^{* 2}<<R T^{*} / v_{m}$, and hence $\tilde{\epsilon}<<1$. Further, we assume $\tilde{M}_{1}>>1$ and in particular we put:

$$
\tilde{M}_{1}=\frac{\tilde{M}}{\epsilon}
$$

where

$$
\tilde{M}=\frac{M_{1} l^{*} \epsilon}{D} \sqrt{\frac{R T^{*}}{v_{m}}} .
$$

In light of the values of $\tilde{M}_{1}$ and $\tilde{\epsilon}$ calculated above for the $\mathrm{Ni}$ - $\mathrm{Cu}$ alloy such assumptions are clearly justified.

The governing equation for the phase field becomes:

$$
\frac{\partial \phi}{\partial \tilde{t}}=\tilde{M}\left(\tilde{\epsilon} \tilde{\nabla}^{2} \phi-\frac{1}{\epsilon} \frac{\partial \tilde{f}}{\partial \phi}\right)
$$

and that for the composition is unchanged from (21). Below we shall consider the limit $\tilde{\epsilon} \rightarrow 0$, which corresponds to considering the situation when the mobility of the phase field is very large and the thickness of the interfaces is very thin. We note that this represents one distinguished limit of the governing equations (21) and (43), which we show below provides a realistic model of phase transitions in isothermal alloys. Other distinguished limits are of course possible, as for instance considered by Caginalp [12], in his analysis of the phase-field model of a pure material.

In the limit $\tilde{\epsilon} \rightarrow 0$ the diffusivity associated with the phase field, which is $\tilde{M} \tilde{\epsilon}$, becomes small, and results in the formation of interior layers within the region $\Omega$ in which the phase field and the concentration vary rapidly, the former from zero to one. Such layers represent solid-liquid interfaces. In this section we seek to derive the jump conditions which relate the concentration field and its derivatives in the vicinity of such layers to their normal velocity 
and curvature. We first consider the outer solution in regions away from the interfacial layers.

Henceforth we only deal with dimensionless quantities in the governing equations, and for simplicity of notation, we omit the tilde's.

\subsection{Outer Solution}

To proceed we seek an outer solution as a regular perturbation in $\epsilon$. Thus we put:

$$
\begin{aligned}
& \phi(\mathbf{x}, t)=\phi^{(0)}(\mathbf{x}, t)+\epsilon \phi^{(1)}(\mathbf{x}, t)+\mathcal{O}\left(\epsilon^{2}\right) \\
& c(\mathbf{x}, t)=c^{(0)}(\mathbf{x}, t)+\epsilon c^{(1)}(\mathbf{x}, t)+\mathcal{O}\left(\epsilon^{2}\right)
\end{aligned}
$$

The leading order problem is

$$
\begin{gathered}
\frac{\partial f}{\partial \phi}\left(\phi^{(0)}, c^{(0)}\right)=0, \\
\frac{\partial c^{(0)}}{\partial t}=\nabla c^{(0)}\left(1-c^{(0)}\right) \cdot \nabla\left(f_{A}-f_{B}\right)+\nabla^{2} c^{(0)},
\end{gathered}
$$

from (46) we find that $\phi^{(0)} \equiv 0$ or $\phi^{(0)} \equiv 1$, depending on whether the region is all liquid or all solid respectively. (The other extremum of $f$ represents an unstable maximum of the energy, and need not be considered.) It follows then from (47) that the leading order solute field simply satisfies the diffusion equation:

$$
\frac{\partial c^{(0)}}{\partial t}=\nabla^{2} c^{(0)}
$$

The governing equations for the first order corrections may also found in a similar way; however, it is easily shown that there are no contributions to $\phi$ beyond the leading order terms, i.e. $\phi$ is exactly zero or one as appropriate.

Because of the generality of the initial data that we have allowed, the initial conditions will not necessarily correspond to $\phi$ zero or unity. In such a situation it is simple to show that there is an initial fast transient, on a time scale $\mathcal{O}(\epsilon)$, in which $\phi$ attains the value zero or unity for almost each $\mathbf{x} \in \Omega$, depending on the value of the initial data for $\phi$ at $\mathbf{x}$. After this short period there will, in general, be large gradients in $\phi$, which represent the interfacial layers. During this short initial period interfaces are created. It is their subsequent development which we now consider. 


\subsection{Inner Solution: The Interfacial Layer}

We assume that the interfacial layer $(\phi(\mathbf{x}, t)=1 / 2)$ is given by the curve $\Gamma(t)$. We introduce a local orthogonal coordinate system $(r(\mathbf{x}, t), s(\mathbf{x}, t))$ where, in the vicinity of $\Gamma(t), r$ measures the normal distance to the curve. Thus $\Gamma(t)$ is therefore described by $r(\mathbf{x}, t)=0$, and $|\nabla r|=1$ in its neighborhood. Further we note that the curvature $\mathcal{K}$ of $\Gamma(t)$, is given by $\mathcal{K}=\nabla^{2} r$. Along $\Gamma(t)$ the coordinate $s(\mathbf{x}, t)$ measures distance from some fixed point. Employing these new independent variables the governing equations become:

$$
\begin{aligned}
\phi_{t}+r_{t} \phi_{r}+s_{t} \phi_{s}= & M\left(\epsilon\left(\phi_{r r}+|\nabla s|^{2} \phi_{s s}+\nabla^{2} r \phi_{r}+\nabla^{2} s \phi_{s}\right)-\epsilon^{-1} \frac{\partial f}{\partial \phi}\right) \\
c_{t}+r_{t} c_{r}+s_{t} c_{s}= & {\left[[c(1-c)]_{r}\left(\frac{\partial f}{\partial c}\right)_{r}+|\nabla s|^{2}[c(1-c)]_{s}\left(\frac{\partial f}{\partial c}\right)_{s}\right.} \\
& +c(1-c))\left\{\left(\frac{\partial f}{\partial c}\right)_{r r}+|\nabla s|^{2}\left(\frac{\partial f}{\partial c}\right)_{s s}+\nabla^{2} r\left(\frac{\partial f}{\partial c}\right)_{r}\right. \\
& \left.\left.+\nabla^{2} s\left(\frac{\partial f}{\partial c}\right)\right\}\right] .
\end{aligned}
$$

In the interfacial layer the rapid changes in the phase-field variable ensure that the effect of the diffusion is important. Thus we scale the coordinate $r$ with $\epsilon$ as:

$$
r=\epsilon \rho .
$$

We also introduce an additional slow time scale, $\tau$ :

$$
\tau=\epsilon t .
$$

We shall employ the method of multiple scales and hence we associate the partial derivative with respect to time in the governing equations with $\partial / \partial t+\epsilon \partial / \partial \tau$.

We expand the dependent variables as:

$$
\bar{c}=\bar{c}^{(0)}(\rho, s, t, \tau)+\epsilon \bar{c}^{(1)}(\rho, s, t, \tau)+\mathcal{O}\left(\epsilon^{2}\right), \bar{\phi}=\bar{\phi}^{(0)}(\rho, s, t, \tau)+\epsilon \bar{\phi}^{(1)}(\rho, s, t, \tau)+\mathcal{O}\left(\epsilon^{2}\right),
$$

where we denote variables in this region by an over bar.

The matching conditions upon the inner solution in the transition region are found by the expanding the outer solution in terms of the inner variables,

$$
\left.\bar{c} \rightarrow c^{(0)}\right|_{\left(r=0^{+}\right)}+\epsilon\left(\left.c^{(1)}\right|_{\left(r=0^{+}\right)}+\left.\rho \frac{\partial c^{(0)}}{\partial r}\right|_{r=0^{+}}\right)+\mathcal{O}\left(\epsilon^{2}\right) \text {, as } \rho \rightarrow \infty
$$




$$
\begin{gathered}
\left.\bar{c} \rightarrow c^{(0)}\right|_{r=0-}+\epsilon\left(\left.c^{(1)}\right|_{r=0-}+\left.\rho \frac{\partial c^{(0)}}{\partial r}\right|_{(r=0-)}\right)+\mathcal{O}\left(\epsilon^{2}\right), \text { as } \rho \rightarrow-\infty, \\
\bar{\phi}=1 \text { as } \rho \rightarrow-\infty \\
\bar{\phi}=0 \text { as } \rho \rightarrow+\infty .
\end{gathered}
$$

In choosing the matching conditions in this way we have assumed that in this local coordinate system the outer liquid region is ahead, and the outer solid region is behind, the interfacial region. To proceed we insert the forms (51), (52) and (53) into the governing equations (49) and (50) to yield a sequence of partial differential equations at each order of $\epsilon$, which we now consider in turn.

\subsubsection{Leading Order}

The leading order problem is:

$$
\begin{aligned}
& r_{t} \bar{\phi}_{\rho}^{(0)}=M\left(\bar{\phi}_{\rho \rho}^{(0)}-f_{\phi}\left(\bar{c}^{(0)}, \bar{\phi}^{(0)}\right)\right), \\
& \left(\bar{c}^{(0)}\left(1-\bar{c}^{(0)}\right) \frac{\partial f_{c}\left(\bar{c}^{(0)}, \bar{\phi}^{(0)}\right)}{\partial \rho}\right)_{\rho}=0 .
\end{aligned}
$$

The latter may be integrated to give that:

$$
\bar{c}^{(0)}\left(1-\bar{c}^{(0)}\right) \frac{\partial f_{c}\left(\bar{c}^{(0)}, \bar{\phi}^{(0)}\right)}{\partial \rho}=\text { function of } s, t \text { and } \tau,
$$

However the matching conditions (54), (55), (56), (57), require that $\partial f_{c} / \partial \rho \rightarrow 0$ as $|\rho| \rightarrow \infty$. Hence we deduce that the function of integration is zero and so:

$$
f_{c}\left(\bar{c}^{(0)}, \bar{\phi}^{(0)}\right)-A(s, t, \tau)=0
$$

where $A$ is a function of integration, which at this stage is undetermined. It is now convenient to define a new modified energy density $F\left(\bar{c}^{(0)}, \bar{\phi}^{(0)}\right)$ by

$$
F\left(\bar{c}^{(0)}, \bar{\phi}^{(0)}\right)=f\left(\bar{c}^{(0)}, \bar{\phi}^{(0)}\right)-A(s, t, \tau) \bar{c}^{(0)}
$$

in which case the equations (58), (59) may be expressed as:

$$
r_{t} \bar{\phi}_{\rho}^{(0)}=M\left(\bar{\phi}_{\rho \rho}^{(0)}-F_{\phi}\left(\bar{c}^{(0)}, \bar{\phi}^{(0)}\right)\right)
$$




$$
F_{c}\left(\bar{c}^{(0)}, \bar{\phi}^{(0)}\right)=0
$$

By employing the matching conditions we find

$$
F_{c}\left(c^{(0)}\left(0^{-}, t\right), 1\right)=F_{c}\left(c^{(0)}\left(0^{+}, t\right), 0\right)=0 .
$$

Equation (64) relates $\bar{c}^{(0)}$ to $\bar{\phi}^{(0)}$ as:

$$
\bar{c}^{(0)}=g_{0}\left(\bar{\phi}^{(0)} ; A\right)
$$

where,

$$
g_{0}\left(\bar{\phi}^{(0)} ; A\right)=\left(1+\exp \left\{f_{B}\left(\bar{\phi}^{(0)}\right)-f_{A}\left(\bar{\phi}^{(0)}\right)-A(s, t, \tau)\right\}\right)^{-1} .
$$

Thus, the modified energy density $F\left(\bar{c}^{(0)}, \bar{\phi}^{(0)}\right) \equiv F\left(g_{0}\left(\bar{\phi}^{(0)} ; A\right), \bar{\phi}^{(0)}\right)$, is a function of $\bar{\phi}^{(0)}$ only which we denote by $E\left(\bar{\phi}^{(0)}\right)\left(\equiv F\left(g_{0}\left(\bar{\phi}^{(0)} ; A\right), \bar{\phi}^{(0)}\right)\right)$, in which case $(63)$ and $(64)$ may be combined as the single equation:

$$
\bar{\phi}_{\rho \rho}^{(0)}-\frac{r_{t}}{M} \bar{\phi}_{\rho}^{(0)}-\frac{d E\left(\bar{\phi}^{(0)}\right)}{d \phi}=0
$$

This problem determines $\bar{\phi}^{(0)}$ (and hence $\bar{c}^{(0)}$ from (66)) and $A(s, t, \tau)$. The function $A(s, t, \tau)$ is found from the asymptotic behaviour required by the matching conditions, which is only possible if the boundary conditions represent singular points of (68), in which case the required solution is the heteroclinic orbit between them. Any other solution would attain the boundary conditions at a finite value of $\rho$.

We now derive an expression for the normal velocity $V_{n}=-r_{t}$. We multiply $(68)$ by $\bar{\phi}_{\rho}^{(0)}$ and integrate to obtain:

$$
\left.\frac{1}{2}\left(\bar{\phi}_{\rho}^{(0)}\right)^{2}\right|_{\rho=-\infty} ^{\rho=\infty}-\frac{r_{t}}{M} \int_{-\infty}^{\infty}\left(\bar{\phi}_{\rho}^{(0)}\right)^{2} d \rho-\left.E\left(\bar{\phi}^{(0)}\right)\right|_{\rho=-\infty} ^{\rho=\infty}=0 .
$$

However from matching:

$$
\left.\bar{\phi}_{\rho}^{(0)}\right|_{\rho=\infty}=\left.\bar{\phi}_{\rho}^{(0)}\right|_{\rho=-\infty}=0,
$$

and

$$
\begin{gathered}
\bar{\phi}^{(0)} \rightarrow 0 \text { as } \rho \rightarrow \infty \\
\bar{\phi}^{(0)} \rightarrow 1 \text { as } \rho \rightarrow-\infty
\end{gathered}
$$


and so:

$$
V_{n}=\frac{M[E(0)-E(1)]}{\Gamma_{0}}
$$

where

$$
\Gamma_{0}=\int_{-\infty}^{\infty}\left(\bar{\phi}_{\rho}^{(0)}\right)^{2} d \rho .
$$

Thus we see that the interface motion is driven by the energy disequilibrium between the two phases; the interface locally advances into the phase with the higher modified energy density, $F\left(\bar{c}^{(0)}, \bar{\phi}^{(0)}\right)$. The interface acts to reduce the energy $F$ of the system by moving to convert regions of high $F$ into low $F$. Time only enters the problem for $\bar{\phi}^{(0)}$ through the matching conditions and hence acts as a parameter. Thus $\bar{\phi}^{(0)}, \bar{c}^{(0)}$ and $V_{n}$ are time dependent and are controlled by the time-dependence of the solute field outside the interfacial layer. In section 4 we show that equations (65) and (73) may be interpreted geometrically as a parallel tangent construction to the Helmholtz free-energy surface for a moving planar interface or a common tangent construction for a planar stationary interface.

\subsubsection{First Order}

We now go on to consider the next order approximation to the governing equations:

$$
\begin{gathered}
\bar{\phi}_{t}^{(0)}+r_{\tau} \bar{\phi}_{\rho}^{(0)}=M\left(\bar{\phi}_{\rho \rho}^{(1)}+M^{-1} V_{n} \bar{\phi}_{\rho}^{(1)}-\left[f_{\phi \phi}\left(\bar{c}^{(0)}, \bar{\phi}^{(0)}\right) \bar{\phi}^{(1)}+f_{c \phi}\left(\bar{c}^{(0)}, \bar{\phi}^{(0)}\right) \bar{c}^{(1)}\right]+\nabla^{2} r \bar{\phi}_{\rho}^{(0)}\right) \\
r_{t} \bar{c}_{\rho}^{(0)}=\left(\bar{c}^{(0)}\left(1-\bar{c}^{(0)}\right)\left(f_{c c}\left(\bar{c}^{(0)}, \bar{\phi}^{(0)}\right) \bar{c}^{(1)}+f_{c \phi}\left(\bar{c}^{(0)}, \bar{\phi}^{(0)}\right) \bar{\phi}^{(1)}\right)_{\rho}\right)_{\rho}
\end{gathered}
$$

We may integrate (76) once from $\rho=-\infty$ to $\rho=+\infty$, which gives:

$$
\left.\left[r_{t} \bar{c}^{(0)}-\bar{c}^{(0)}\left(1-\bar{c}^{(0)}\right)\left(f_{c c} \bar{c}^{(1)}+f_{c \phi} \bar{\phi}^{(1)}\right)_{\rho}\right]\right|_{\rho=\infty} ^{\rho=\infty}=0 .
$$

On employing the matching conditions this gives that:

$$
\left(\left.\frac{\partial c^{(0)}}{\partial r}\right|_{0^{+}}-\left.\frac{\partial c^{(0)}}{\partial r}\right|_{0^{-}}\right)=-V_{n}\left(\left.c^{(0)}\right|_{\left(r=0^{+}\right)}-\left.c^{(0)}\right|_{\left(r=0^{-}\right)}\right) .
$$

This simply represents a conservation of solute normal to the moving interface and is the usual condition employed in the classical model model of phase transitions involving sharp interfaces. 
As an isolated system evolves, it approaches its final minimum energy, in which case the energy differences across the interface, which drive the interfacial motion, become small. Thus $V_{n} \rightarrow 0$ as $t \rightarrow \infty$ and so the system evolves on a slower time scale. To determine the interfacial dynamics on this long time scale, which is given by the scaled time $\tau$, we put $V_{n}=0$, in which case the interfacial velocity is given by $r_{\tau}=\bar{V}_{n}$. In this regime the concentration gradients near the interfaces are small, $\mathcal{O}(\epsilon)$. The slow velocity $\bar{V}_{n}$ is given from a solvability condition that arises from the first order problem. In the Appendix we discuss the adjoint problem and use it to determine the solvability condition, which is:

$$
c^{(1)}\left(0^{+}\right)\left(\frac{c^{(0)}\left(0^{+}\right)-c^{(0)}\left(0^{-}\right)}{c^{(0)}\left(0^{-}\right)\left(1-c^{(0)}\left(0^{+}\right)\right)}\right)+\Gamma_{0}\left(M^{-1} \bar{V}_{n}+\mathcal{K}\right)=0,
$$

where $\Gamma_{0}$ is given by equation (74). The above solvability condition determines the slow component of the normal interface velocity, $\bar{V}_{n}$, which is thus dependent upon the local curvature, $\mathcal{K}$, and the perturbed concentration in the liquid, $c^{(1)}\left(0^{+}\right)$. In the Appendix we also derive another solvability condition, equation (116), which relates the perturbed interfacial concentrations $c^{(1)}\left(0^{-}\right)$and $c^{(1)}\left(0^{+}\right)$at the interface.

\subsubsection{Temporal Evolution}

From the above analysis we can identify three different stages in the evolution of the solution of the phase-field equations based on initial data in which $0 \leq c_{\text {init }}, \phi_{i n i t} \leq 1$ :

- Stage I. Initially there is a very fast initial transient $\mathcal{O}(\epsilon)$ in which the phase field adjusts locally to the values zero or unity. We interpret this as a period in which interfacial layers are born and regions of solid and liquid are differentiated.

- Stage II. Subsequently, on an $\mathcal{O}(1)$ time scale, away from these interfacial layers in the outer regions, $\phi$ is either zero or unity and the solute concentration is governed by the diffusion equation. Between the outer regions the interfacial layers exist. The local normal velocity of these interfaces is determined by the local jump in the modified energy density across the layer, $E$, (and thus by the deviation of the composition from the equilibrium values) described by (73). More specifically, the interfaces move so as to decrease the energy of the system. The interfacial motion will slow down as the system approaches its minimum energy, i.e. $V_{n} \rightarrow 0$ as $t \rightarrow \infty$. 
- Stage III. Finally, on a longer time scale $\mathcal{O}\left(\epsilon^{-1}\right)$, the normal interfacial velocity is given from (79). The slow motion of the interface is controlled by a balance between curvature and thermodynamic disequilibrium, the latter is represented by the term involving the perturbed concentration. This represents the process of Ostwald ripening.

Thus, initially the system evolves very fast to minimise its energy, by evolving the phase field at each point in space to zero or unity; the minima of the energy density for a fixed concentration. Subsequently diffusion acts and the interfaces move to reduce the energy differences across them. Finally they slowly evolve in response to local energy disequilibrium and kinetic effects, as well as to reduce the energy associated with their curvature.

\subsubsection{A Mechanical Analogy}

The leading order behaviour, discussed above is described by the system:

$$
\begin{gathered}
\frac{d^{2} y}{d t^{2}}+M^{-1} V_{n} \frac{d y}{d t}=F_{y}(x, y), \\
0=F_{x}(x, y),
\end{gathered}
$$

when we identify $x$ with $\bar{c}^{(0)}, y$ with $\bar{\phi}^{(0)}, \rho$ with time, and $F(x, y)=f(x, y)-A x$ as before, where $\mathrm{A}$ is to be determined and $f$ is the free energy density. This system represents the motion of a particle in a potential $z=-F(x, y)$, constrained to lie on the curve $x=$ $g_{0}(y ; A)$, with friction acting only in the y direction. We note that the surface $z=F(x, y)$ is obtained from that of the free energy density surface $z=f(x, y)$ by inverting it and tilting it in the $x\left(\bar{c}^{(0)}\right)$ direction; the amount of tilt depending on the value of $A$. The frictional constant is equal to $M^{-1} V_{n}$, and so the stationary interface corresponds to the frictionless case. The matching conditions require that the particle follows a path such that it approaches a stationary state as time goes to plus and minus infinity. The only possible such motion is one that begins and ends at local maxima of the surface $z=-F(x, y)$. The form of the free energy density ensures that such local maxima only occur for one given value of $x\left(\bar{c}^{(0)}\right)$ on $y=0\left(\bar{\phi}^{(0)}=0\right)$ and one given value of $x\left(\bar{c}^{(0)}\right)$ on $y=1\left(\bar{\phi}^{(0)}=1\right)$, both of which depend on $\mathrm{A}$ and lie on the curve $x=g_{0}(y ; A)$. Thus the particle must follow a trajectory between these local maxima which traverses from $y=0$ to $y=1$. When the interface is stationary, 
the particle moves in the absence of friction, in which case the constant $A$ (or equivalently the amount of tilt of the surface) is chosen such that the heights of the two maxima are the same. For a non-stationary interface the constant $A$ must be chosen such that the potential energy difference associated with the difference in height of the two maxima is just sufficient to account for the energy dissipated by friction. A mechanical analogy was noted by Cahn [4] for the Cahn-Hilliard equation, as well as by Harrowell and Oxtoby [20].

\subsection{Discussion Of Asymptotics}

As shown in section 3.1 the governing equation for the solute reduces to the diffusion equation in the solid and liquid regions with the diffusivity $D$ given by $D=M_{1} R T^{*} / v_{m}$. Thus the governing equation for composition given here is the same as those of a sharp interface, or classical model, in the solid and liquid regions.

We see from (73) that the temperature, $T$, and normal velocity, $V_{n}$, determine the leading order interfacial concentrations in the solid and liquid. Thus $c_{S}=H_{S}\left(T, V_{n}\right)$ and $c_{L}=H_{L}\left(T, V_{n}\right)$. When the interface is stationary, $V_{n}=0$, then the functions $c_{S}=H_{S}(T, 0)$ and $c_{L}=H_{L}(T, 0)$ simply represent the solidus and liquidus curves of the phase diagram of the alloy (see section 4.1).

The asymptotic analysis generalises the results given in section 2.1 for the interface kinetics and surface energy of a pure material to the case of an alloy. In particular (73) is the non-dimensional analogue of (31) for the kinetics of a pure material. The solvability condition (79) represents an energy balance at $\mathcal{O}(\epsilon)$; the first two terms are the change in energy due to the perturbed concentration and interfacial motion, the last term represents the surface energy. Thus the surface energy, $\sigma$, is $\epsilon \int_{-\infty}^{\infty}\left(\bar{\phi}_{\rho}^{(0)}\right)^{2} d \rho$ which may be rewritten with the help of (69) as

$$
\sigma=\epsilon \sqrt{2} \int_{0}^{1} \sqrt{E(\phi)} d \phi
$$

which is the generalisation of (27) and a similar result of Cahn and Hilliard [4]. However, for the alloy these expressions for the surface energy (82) and interface kinetics (73) have no simple analytic form as they do for the case of the pure material. For simplicity we therefore choose to determine the parameters of the phase-field model in the way discussed in section 
2.2 by making contact with the properties of the pure components.

\section{Constant Velocity Planar Solutions as $\epsilon \rightarrow 0$.}

In this section we look for solutions in the limit $\epsilon \rightarrow 0$ corresponding to a planar interface advancing at at constant normal velocity $\mathrm{V}$. We only consider the outer solution. We take a coordinate coincident with the moving interface, which is then given by the plane $z=0$. The leading order problem for $c$ is then:

$$
\begin{gathered}
c_{z z}+V c_{z}=0 \\
V=\frac{M[E(0)-E(1)]}{\Gamma_{0}} \\
\left.c\right|_{z=0^{-}}=g_{0}(1 ; A),\left.\quad c\right|_{z=0^{+}}=g_{0}(0 ; A) \\
\left(\left.\frac{\partial c}{\partial r}\right|_{z=0^{+}}-\left.\frac{\partial c}{\partial r}\right|_{z=0^{-}}\right)=-V\left(\left.c\right|_{z=0^{+}}-\left.c\right|_{z=0^{-}}\right)
\end{gathered}
$$

with $c(z) \rightarrow c_{\infty}$ as $|z| \rightarrow \infty$. The leading order solution is easily found to be:

$$
\begin{gathered}
c(z)=c_{S}+\left(c_{L}-c_{S}\right) \exp (-V z), z>0, \\
c(z)=c_{S}, z<0
\end{gathered}
$$

where we associate $c_{S}$ and $c_{L}$ with $\left.c^{(0)}\right|_{z=0-}$ and $\left.c^{(0)}\right|_{z=0+}$ respectively, which along with the constant $A$ are determined by the three conditions given in (84) and (85). We note that $c_{\infty}=c_{S}$, which therefore cannot be prescribed independently of the temperature, $T$.

The segregation coefficient is

$$
k=\frac{c_{S}}{c_{L}},
$$

which depends on the interface speed V. For this reason we first consider the situation when the interface is stationary.

\subsection{Stationary Interface: $V=0$}

In this case the concentration is constant in each phase:

$$
c(z)=c_{S}, z<0, c(z)=c_{L}, z>0
$$


where $c_{S}, c_{L}$, and $A$ satisfy the three conditions in (84) and (85) with $\mathrm{V}=0$. The condition (84) gives that:

$$
F\left(c_{S}, 1\right)=F\left(c_{L}, 0\right) .
$$

We note that from the definition of the function $g_{0}$ given by (66) and the conditions in (85) that:

$$
F_{c}\left(c_{S}, 1\right)=F_{c}\left(c_{L}, 0\right)=0 .
$$

Using the fact that $F(c, \phi)=f(c, \phi)-A c$ it is easily shown that these statements are equivalent to requiring that $c_{S}$ and $c_{L}$ are chosen such there is a common tangent between the curves $f(c, 0)$ and $f(c, 1)$. This construction, see Figure $3(a)$, is precisely that given by classical thermodynamics to determine the equilibrium concentrations in this situation. It is usually derived by requiring that the chemical potential of the two phases on either side of the interface in a first order phase transition is continuous. The locus of pairs of compositions $c_{S}$ and $c_{L}$ at different temperatures provides the liquidus and solidus curves of the phase diagram. The common tangent construction also corresponds to constructing a common tangent plane to the free energy surface, shown in Figure 2, which is tangent to both sections of the energy surfaces, $\phi=0$ and $\phi=1$.

We now employ this stationary interface solution to examine the possible stationary configurations of the system as $\epsilon \rightarrow 0$. In particular we consider a one dimensional box, of length $L$, whose ends are impermeable to both components of the solution. The average composition $C$ is $\frac{1}{L} \int_{0}^{L} c d x$. There are single phase solutions; single phase solid $(\phi \equiv 1)$ and single phase liquid ( $\phi \equiv 0)$. These exist for all values of the average composition, $C \in[0,1]$, and are linearly stable. However, the analysis above indicates that other solutions are possible representing a single interface between two regions of different phase when $\min \left(c_{S}, c_{L}\right)<$ $C<\max \left(c_{S}, c_{L}\right)$. In Figure 4 we schematically illustrate the possible solutions. The variation of the fraction solid is given classically (disregarding the interfacial surface energy) by the "lever" rule and is linear in the composition between $c_{S}$ and $c_{L}$. 


\subsection{Non-Stationary Interface $V \neq 0$}

In this situation the only condition that is different is (91) which becomes:

$$
F\left(c_{L}, 0\right)-F\left(c_{S}, 1\right)=M^{-1} V \Gamma_{0},
$$

the left hand side of which is a constant, $\Delta F(V, T)$, for a given interface velocity, $V$, and temperature, $T$. The conditions (92), (93) are equivalent to selecting $c_{S}$ and $c_{L}$ such that their tangents on the curves $f(c, 0)$ and $f(c, 1)$ are parallel, but the tangents are a vertical distance $\Delta F(V, T)\left(=F\left(c_{L}, 0\right)-F\left(c_{S}, 1\right)\right)$ apart, see Figure 3(b). It follows that the equilibrium concentrations of a moving planar interface are dependent on its normal velocity.

From (84) and (85) we find that the segregation coefficient is given by:

$$
k=\exp \left(-\Delta F(V, T)-f_{B}(1)+f_{B}(0)\right),
$$

which for small $V$ is given approximately by:

$$
k=k_{0}\left(1-\left.V M^{-1} \Gamma_{0}\right|_{(V=0)}+\mathcal{O}\left(V^{2}\right)\right),
$$

where $k_{0}$ is the value of the segregation coefficient for the stationary planar interface. Similarly for a small energy difference across the interface the segregation coefficient may be approximated by:

$$
k=k_{0}\left[1-\Delta F(0, T)+\mathcal{O}\left((\Delta F(0, T))^{2}\right)\right] .
$$

From equation (68) we numerically computed $c_{L}, c_{S}$ and $k$ as a function of the interface velocity. The results are displayed in Figure 5. We see that the solid-liquid interfacial concentrations and the segregation coefficient are monotonically decreasing functions of the interface velocity, (the latter dependence is in agreement with the small growth rate expression for $k,(95))$.

Experimentally, the behaviour of the segregation coefficient $k$ with increasing interface velocity has been shown to change from the equilibrium value (normally less than unity) at low velocities to values near unity for high velocities $(\approx 1 \mathrm{~m} / \mathrm{s})$, see White et al. [21]. This tendency towards unity is not recovered in the current model. We anticipate that inclusion of a term in the energy functional involving the solute gradient energy in addition to the phase field gradient energy will produce a wide variety of possible behaviour, depending on 
the relative importance of the two gradient energy terms. In fact a model by Baker [23] shows that $k$ need not change monotonically towards unity with increasing velocity.

\section{Numerical Calculations}

In this section we give the results of numerical calculations upon our model. The aim of these calculations is two-fold, first, to verify as far as possible the asymptotic analysis given in the previous section, and second to demonstrate that our model qualitatively models the behaviour of phase transitions in an isothermal alloy. In order to apply our model to situations in which the interface is sharp, we require the value of $\epsilon$ to be small. This provides the major difficulty in conducting calculations, which is that the interfacial layers are necessarily thin, and thus a sufficiently fine computational mesh is required in order to resolve the solution structure within them. For this reason it is much easier to conduct calculations in one spatial dimension than two. Thus, in this section we first employ calculations in one dimension to verify quantitatively much of the boundary layer analysis, and we further perform two-dimensional calculations with a somewhat larger value of $\epsilon$ to provide evidence that our model is qualitatively in agreement with this analysis and that it shows behaviour similar to that found in real systems.

\subsection{One-Dimensional Calculations}

We consider the evolution of the system (20) and (21) in one spatial dimension, $x$, on the domain $0<x<L$. We imposed the boundary conditions $\phi_{x}=c_{x}=0$ at $x=0$, and $\phi_{x}=0, c_{x}+\alpha_{0} c=\alpha_{1}$ at $x=L$. The initial data is given by $\phi(x, 0)=\phi_{0}(x), c(x, 0)=c_{0}(x)$. We used the software package PDECOL [24], which employs a finite element collocation procedure for the spatial discretisation and the method of lines to advance the solution in time. In all cases we used 500 break points. The first set of calculations were aimed to test the asymptotic analysis by generating the planar traveling wave solutions predicted by the theory given in section 4 . The most trivial solution corresponds to the stationary interface, in which case the asymptotic analysis predicts the (constant) values of the concentration either side of the interface. With initial data for $c$ and $\phi$ set to the appropriate step functions, 
it is found that the predicted concentrations were indeed recovered within the accuracy of the scheme, with $\epsilon$ taken sufficiently small. We also conducted calculations for a nonstationary interface. The results of the asymptotic analysis displayed in Figure 5 predict the interface velocity, $V$ to be 0.1 when $c_{S}=2.0339 \times 10^{-2}$ and $c_{L}=0.3679$. In order to avoid end effects we chose $\alpha_{0}=V$ and $\alpha_{1}=c_{s}$, which is exactly satisfied by the traveling wave solution concentration profile, (87), when $\phi=0$. Physically this corresponds to extracting solute from the liquid phase at the boundary of the domain in order to maintain the supersaturation which is driving the interfacial motion. We put $\beta_{A}=-0.1, \beta_{B}=0.1$, $W_{A}=W_{B}=1.0, \epsilon=10^{-3}$, and $L=0.4$. The initial data was $\phi_{0}=0.1+0.9 H(x-0.04)$ and $c_{0}=c_{S}+H(x-0.04)\left(c_{L}-c_{S}\right) \exp [-V(x-0.04)]$, where $H(x)$ is the Heaviside function. In Figure 6 we display the computed composition profile at times of 0.0, 1.0, 2.0 and 3.0. It clearly reproduces the predicted traveling wave solution with the predicted constant velocity interface velocity of 0.1 . We note that initially the velocity of the interface is slightly less than that predicted by the asymptotic analysis as $\epsilon \rightarrow 0$. We attribute this to the adjustment required by the profile in the interfacial boundary layer, which was not properly given by the initial data. This is manifested by the smoothing out of the initial discontinuity of the initial data in this region. The above calculations quantitatively verify the result given by (73) and confirm that the modified energy differences drive the interfacial motion.

We also conducted a numerical calculation which models the motion of a planar interface in a closed system in response to the presence of an initially supersaturated liquid. From Figure 5 the interface is stationary when $c_{S}=0.1589$ and $c_{L}=0.8411$. We chose the same parameters as the previous calculation but the initial data for the composition was different: $c_{0}=c_{S}+\left(0.6-c_{S}\right) H(x-0.04)$. Also the boundary condition for the composition at $x=0.4$ was $c_{x}=0$. This initial state corresponds to a supersaturated liquid phase with composition $0.6\left(<c_{L}\right)$. In Figure 7 we display the composition profile at times $0,2.0,4.0,6.0,8.0$ and 10.0. There is in fact a rapid initial transient, not apparent in this figure, in which the concentration in the solid decreases to approximately 0.1 and the concentration in the liquid adjacent to the interface increases. Subsequently, as shown in Figure 7 the concentration boundary layer so formed in the liquid is accompanied by forward interfacial motion. Finally, the system relaxes back to the uniform concentrations predicted by the asymptotic theory 
for a stationary interface. This behaviour is expected on physical grounds; the interface moves to relieve the supersaturation subject to the constraint that the composition of the system is conserved. However, in a real system it would be expected that the planar interface would be unstable and evolve to a cellular structure as it moved forward. We next consider two-dimensional calculations.

\subsection{Two-Dimensional Calculations}

As discussed above, the thin interfacial layers presently cause a difficulty in conducting accurate numerical calculations. In two dimensions this problem is important because the computer storage and processor requirements associated with fine meshes become restrictive. Below we present the results of preliminary calculations which employ a uniform $80 \times 80$ mesh, the finest mesh that was feasible using the computer facilities available to us. We used the software package PDETWO. This employs second order accurate central finite differences to conduct the spatial discretisation, allied to an accurate ordinary differential integrator to evolve the solution in time. We have performed a number of calculations. Below we show typical results for one particular case.

We performed a calculation in a square region of side unity. The initial data for the phase field and composition was generated from a random distribution of these variables with amplitude 0.01 about a mean value of a half. The following values were used for the various nondimensional constants; $\beta_{A}=-0.1, \beta_{B}=0.1, W_{A}=10, W_{B}=10, M_{1}=$ 40 , and $\epsilon=\frac{1}{40}$. These values for $M_{1}$ and $\epsilon$ are chosen to lie in the asymptotic regime of our analysis. In particular the size of $\epsilon$ is a compromise between making it sufficiently small that the interfacial layers are thin enough for the asymptotic analysis to hold but large enough that they are adequately resolved by the finite difference grid. In Figure 8 we display the phase field at times $t=0,0.01,0.05,0.1$ and 1 .

From Figures 8(a-d), it is clear there is a rapid initial transient evolution of the phase field to $\phi=0$ or 1 , which is complete by $t=0.1$ when fully differentiated regions of solid and liquid have been established. This is in agreement with our asymptotic analysis although it is evident that there is also a significant coarsening of the solid regions during this period. Subsequently this coarsening behaviour continues until $t=1$, the final time displayed. 
We monitored the total amount of solute present as a function of time. It was found that our present numerical scheme suffers a small loss of solute from the system. The loss is most pronounced at small times when the system is evolving most rapidly. A total loss of $1.26 \%$ by $t=1$ is observed. The lack of solute conservation in the numerical scheme precludes longtime integration of the governing equations, since the loss can be large enough to eventually shift the average solute concentration $C$ to values outside the range $c_{S}<C<c_{L}$ for the two-phase system. This results in eventual dissolution of one of the phases, leading to a final state consisting of a single phase. The final period of evolution that is predicted by the asymptotic analysis, in which curvature variations are important, is thus difficult to observe with the current numerical scheme.

The qualitative behaviour observed largely substantiates our asymptotic analysis, demonstrating the rapid period of interfacial genesis and order-one motion due to energy differences. In addition, coarsening is observed which is reminiscent of Ostwald ripening.

\section{Conclusions}

In this paper we have presented a new phase-field model which describes the spatial and temporal evolution of isothermal phase transitions between ideal binary alloy solution phases. We have shown how the parameters which characterise this phase-field model are related to material parameters and thermodynamics data employed in classical sharp interface models.

We conducted an asymptotic analysis of the governing equations in the distinguished limit $\epsilon \rightarrow 0$ with $M_{1}=\mathcal{O}\left(\epsilon^{-1}\right)$, which approximates the situation when the mobility of the phase field is large and its diffusivity small. In this regime there exist thin interfacial layers of width $\mathcal{O}(\epsilon)$, and so we recovered a sharp interface model in this limit. From this analysis we determined the interfacial dynamics and showed that the interface evolved on three time scales. There is short initial transient in which interfaces are born. It results primarily from the response of the system to the initial data we have chosen and does not necessarily represent the process of nucleation. This is followed by evolution on the diffusion time scale in which the interfacial motion is controlled by the energy difference across the interface and diffusion in the solid and liquid phases. Finally there is a long period in which the interfaces 
move slowly in response to their surface energy and thermodynamic disequilibrium. This final period we interpret as representing Ostwald ripening. We constructed planar interface solutions in this asymptotic limit and were able to characterise the interface kinetics in terms of the thermodynamic driving force and show a dependence of the segregation coefficient on interfacial velocity.

Lastly, we presented numerical calculations which illustrate the predictions of our asymptotic analysis.

It is clear from this work that this phase-field model provides a good description of isothermal phase transitions between binary solution phases and provides a first step in devising a non-isothermal phase-field model for an alloy system.

\section{Acknowledgements}

The authors are grateful to G. Caginalp, J. W. Cahn, S. R. Coriell, R. Kobayashi, B. T. Murray, and R. F. Sekerka for many helpful discussions. AAW acknowledges support by the Science and Engineering Research Council of Great Britain, and the Royal Society of London. GBM acknowledges support by the Microgravity Science and Applications Division, NASA, and the Applied and Computational Mathematics Program, DARPA. AAW and GBM were supported in part by a NATO Collaborative Research Grant.

\section{Appendix: The Adjoint Problem}

In order to obtain equation (79), representing the slow time evolution of a curved interface, which arises from solvability conditions on the first order equations (75) and (76) we require their adjoint solution when $V_{n}=0$. Equations (75) and (76) when $V_{n}=0$ are:

$$
\begin{gathered}
\bar{\phi}_{\rho \rho}^{(1)}-\left[f_{\phi \phi}\left(\bar{c}^{(0)}, \bar{\phi}^{(0)}\right) \bar{\phi}^{(1)}+f_{c \phi}\left(\bar{c}^{(0)}, \bar{\phi}^{(0)}\right) \bar{c}^{(1)}\right] \\
=M^{-1}\left[\bar{\phi}_{t}^{(0)}+r_{\tau} \bar{\phi}_{\rho}^{(0)}-M \nabla^{2} r \bar{\phi}_{\rho}^{(0)}\right], \\
{\left[\bar{c}^{(0)}\left(1-\bar{c}^{(0)}\right)\left(f_{c c}\left(\bar{c}^{(0)}, \bar{\phi}^{(0)}\right) \bar{c}^{(1)}+f_{c \phi}\left(\bar{c}^{(0)}, \bar{\phi}^{(0)}\right) \bar{\phi}^{(1)}\right)_{\rho}\right]_{\rho}=0 .}
\end{gathered}
$$


with

$$
\begin{gathered}
\left.\bar{c}^{(1)} \rightarrow c^{(1)}\right|_{\left(r=0^{+}\right)} \text {as } \rho \rightarrow \infty, \\
\left.\bar{c}^{(1)} \rightarrow c^{(1)}\right|_{\left(r=0^{-}\right)} \text {as } \rho \rightarrow-\infty,
\end{gathered}
$$

We introduce the adjoint solution $\left(\phi^{+}, c^{+}\right)$and take the inner product of (97) and (98) with the adjoint solution. This gives

$$
\begin{gathered}
\int_{-\infty}^{\infty} M^{-1}\left[\bar{\phi}_{t}^{(0)}+r_{\tau} \bar{\phi}_{\rho}^{(0)}-M \nabla^{2} r \bar{\phi}_{\rho}^{(0)}\right] \phi^{+} d \rho= \\
{\left.\left[\bar{c}^{(0)}\left(1-\bar{c}^{(0)}\right)\left(f_{c c} \bar{c}^{(1)}+f_{c \phi} \bar{\phi}^{(1)}\right)_{\rho} c^{+}-\bar{c}^{(0)}\left(1-\bar{c}^{(0)}\right)\left(f_{c c} \bar{c}^{(1)}+f_{c \phi} \bar{\phi}^{(1)}\right) c_{\rho}^{+}\right]\right|_{-\infty} ^{\infty}+} \\
\int_{-\infty}^{\infty}\left\{\bar{\phi}^{(1)}\left[\phi_{\rho \rho}^{+}-f_{\phi \phi} \phi^{+}+f_{c \phi}\left(\bar{c}^{(0)}\left(1-\bar{c}^{(0)}\right) c_{\rho}^{+}\right)_{\rho}\right]+\bar{c}^{(1)}\left[\left(\bar{c}^{(0)}\left(1-\bar{c}^{(0)}\right) c_{\rho}^{+}\right)_{\rho} f_{c c}-f_{c \phi} \phi^{+}\right]\right\} d \rho+ \\
{\left.\left[\bar{\phi}_{\rho}^{(1)} \phi^{+}-\bar{\phi}^{(1)} \phi_{\rho}^{+}\right]\right|_{-\infty} ^{\infty}}
\end{gathered}
$$

Thus the adjoint problem is:

$$
\begin{aligned}
\phi_{\rho \rho}^{+}-f_{\phi \phi} \phi^{+}+f_{c \phi}\left(\bar{c}^{(0)}\left(1-\bar{c}^{(0)}\right) c_{\rho}^{+}\right)_{\rho} & =0 \\
\left(\bar{c}^{(0)}\left(1-\bar{c}^{(0)}\right) c_{\rho}^{+}\right)_{\rho} f_{c c}-f_{c \phi} \phi^{+} & =0 .
\end{aligned}
$$

This has a solution:

$$
\begin{gathered}
c^{+}(\rho)=\int_{0}^{\rho} \frac{B_{1}-B_{2} \bar{c}^{(0)}(x)}{\bar{c}^{(0)}\left(1-\bar{c}^{(0)}(x)\right)} d x, \\
\phi^{+}(\rho)=B_{2} \bar{\phi}_{\rho}^{(0)}(\rho)
\end{gathered}
$$

where $B_{1}$ and $B_{2}$ are constants of integration. We note that $c^{+} \sim \alpha_{+} \rho+\beta_{+}+o(1)$, as $\rho \rightarrow+\infty$ and $c^{+} \sim \alpha_{-} \rho+\beta_{-}+o(1)$, as $\rho \rightarrow-\infty$. The constants $\alpha_{+}, \alpha_{-}, \beta_{+}, \beta_{-}$are determined by the constants of integration as:

$$
\begin{gathered}
\alpha_{+}=\frac{B_{1}}{\left.c^{(0)}\right|_{\left(r=0^{+}\right)}\left(1-\left.c^{(0)}\right|_{\left(r=0^{+}\right)}\right)}-\frac{B_{2}}{1-\left.c^{(0)}\right|_{\left(r=0^{+}\right)}} \\
\alpha_{-}=\frac{B_{1}}{\left.c^{(0)}\right|_{\left(r=0^{-}\right)}\left(1-\left.c^{(0)}\right|_{\left(r=0^{-}\right)}\right)}-\frac{B_{2}}{1-\left.c^{(0)}\right|_{\left(r=0^{-}\right)}} \\
\beta_{-}=B_{1} \gamma_{1}^{-}+B_{2} \gamma_{2}^{-}, \\
\beta_{+}=B_{1} \gamma_{1}^{+}+B_{2} \gamma_{2}^{+},
\end{gathered}
$$


where

$$
\begin{gathered}
\gamma_{1}^{-}=\int_{0}^{-\infty}\left[\frac{1}{\bar{c}^{(0)}\left(1-\bar{c}^{(0)}\right)}-\frac{1}{\left.c^{(0)}\right|_{\left(r=0^{-}\right)}\left(1-\left.c^{(0)}\right|_{\left(r=0^{-}\right)}\right)}\right] d \rho, \\
\gamma_{1}^{+}=\int_{0}^{+\infty}\left[\frac{1}{\bar{c}(0)\left(1-\bar{c}^{(0)}\right)}-\frac{1}{\left.c^{(0)}\right|_{\left(r=0^{+}\right)}\left(1-\left.c^{(0)}\right|_{\left(r=0^{+}\right)}\right)}\right] d \rho, \\
\gamma_{2}^{-}=\int_{0}^{-\infty}\left[\frac{1}{\left(1-\bar{c}^{(0)}\right)}-\frac{1}{\left(1-\left.c^{(0)}\right|_{\left(r=0^{-}\right)}\right)}\right] d \rho, \\
\gamma_{2}^{+}=\int_{0}^{+\infty}\left[\frac{1}{\left(1-\bar{c}^{(0)}\right)}-\frac{1}{\left(1-\left.c^{(0)}\right|_{\left(r=0^{+}\right)}\right)}\right] d \rho .
\end{gathered}
$$

Inserting these forms into the inner product (101) gives

$$
\begin{aligned}
B_{1}\left[\frac{c^{(1)}\left(0^{-}\right)}{c^{(0)}\left(0^{-}\right)\left(1-c^{(0)}\left(0^{-}\right)\right)}\right. & \left.-\frac{c^{(1)}\left(0^{+}\right)}{c^{(0)}\left(0^{+}\right)\left(1-c^{(0)}\left(0^{+}\right)\right)}\right] \\
& +B_{2}\left[\frac{c^{(1)}\left(0^{+}\right)}{1-c^{(0)}\left(0^{+}\right)}-\frac{c^{(1)}\left(0^{-}\right)}{1-c^{(0)}\left(0^{-}\right)}\right] \\
& =B_{2} \int_{0}^{+\infty} M^{-1}\left(r_{\tau} \bar{\phi}_{\rho}^{(0)}-M \nabla^{2} r \bar{\phi}_{\rho}^{(0)}\right) \bar{\phi}_{\rho}^{(0)} d \rho .
\end{aligned}
$$

This is true for all choices of the constants $B_{1}$ and $B_{2}$ and so we equate the coefficients of these quantities in the above expression to obtain the following two nontrivial solvability conditions:

$$
\frac{c^{(1)}\left(0^{-}\right)}{c^{(0)}\left(0^{-}\right)\left(1-c^{(0)}\left(0^{+}\right)\right)}=\frac{c^{(1)}\left(0^{+}\right)}{c^{(0)}\left(0^{+}\right)\left(1-c^{(0)}\left(0^{-}\right)\right)}
$$

and

$$
\frac{c^{(1)}\left(0^{+}\right)}{1-c^{(0)}\left(0^{+}\right)}-\frac{c^{(1)}\left(0^{-}\right)}{1-c^{(0)}\left(0^{-}\right)}=\int_{-\infty}^{+\infty}\left(M^{-1} r_{\tau}-\nabla^{2} r\right)\left(\bar{\phi}_{\rho}^{(0)}\right)^{2} d \rho .
$$

These solvability conditions may be combined to give:

$$
c^{(1)}\left(0^{+}\right)\left(\frac{c^{(0)}\left(0^{+}\right)-c^{(0)}\left(0^{-}\right)}{c^{(0)}\left(0^{+}\right)\left(1-c^{(0)}\left(0^{+}\right)\right)}\right)=-\Gamma_{0}\left(\mathcal{K}+M^{-1} \bar{V}_{n}\right),
$$

where $\bar{V}_{n}=-r_{\tau}$ is the the slowly varying component of the normal velocity of the interface, $\mathcal{K}=\nabla^{2} r$ is the mean curvature of the interface, and

$$
\Gamma_{0}=\int_{-\infty}^{\infty}\left(\bar{\phi}_{\rho}^{(0)}\right)^{2} d \rho .
$$




\section{References}

[1] S.-S. Huang and M. E. Glicksman, Acta Metallurgica 29, 701 (1981).

[2] S. C. Hardy, Phil. Mag. 35, 471 (1977).

[3] L. D. Landau and I. M. Khalatnikov, Dokl. Akad. Nauk SSSR 96, 469 (1954). English translation in: Collected works of L. D. Landau, ed. D. ter Haar (Pergamon, Oxford, 1965) pp $626-633$.

[4] J. W. Cahn and J. E. Hilliard, J. Chem. Phys. 28, 258 (1958).

[5] J. W. Cahn and J. E. Hilliard, J. Chem. Phys. 31, 688 (1959).

[6] J. W. Cahn, Acta Metallugica 9, 795 (1961).

[7] J. S. Langer and R. F. Sekerka, Acta Met. 23, 1225 (1975).

[8] B. I. Halperin, P. C. Hohenburg, and S.-K. Ma, Phys. Rev. B. 10, 139 (1974).

[9] J. S. Langer, in: Directions in Condensed Matter Physics (World Science Publishers, 1986) pp 164-186.

[10] G. Fix, in: Free Boundary problems, ed. A. Fasano and M. Primocerio, (Pitman, London, 1983) pp. 580-589.

[11] G. Caginalp, Arch. Rat. Mech. Anal. 92, 205 (1986).

[12] G. Caginalp, Phys. Rev. A. 39, 5887 (1989).

[13] G. Caginalp and P. C. Fife, Phys. Rev. B. 33, 7792 (1986).

[14] J. Smith, J. Comp. Phys. 39, 112 (1981).

[15] G. Caginalp and E. A. Socolowski, J. Comp. Phys. 95, 85 (1991).

[16] R. Kobayashi, private communication (1990).

[17] O. Penrose and P. C. Fife, Physical D 43, 44 (1990). 
[18] P. C. Fife, in Proc. Taniguchi Int. Symp. on Nonlinear PDEs and Applications, (Kinokuniya Pub. Co., 1990).

[19] S. M. Allen and J. W. Cahn, Acta Met. 27, 1085 (1979).

[20] P. R. Harowell and D. W. Oxtoby, J. Chem Phys. 86, 2932 (1987).

[21] C. W. White, B. R. Appleton, D. Stritzer, D. M. Zehner and S. R. Wilson, Mat. Res. Soc. Symp. 1, 59 (1981).

[22] S. R. Coriell and D. Turnbull, Acta Met. 30, 2135 (1982).

[23] J. C. Baker, Interfacial partitioning during solidification, Ph D thesis, MIT (1970). See also J. W. Cahn, S. R. Coriell, and W. J. Boettinger, Rapid solidification. Laser and electron beam processing of materials (Academic Press, 1980).

[24] N. K. Madsen and R. F. Sincovec, ACM-TOMS 5, 326 (1979). 


\section{Captions to the Figures}

Figure 1. The free energy density $f(\phi, T)$, employed by Kobayashi [16] displayed as a function of $\phi$ for three values of the temperature near the melting temperature, $T_{M}$ : for $T<T_{M}$ $(\beta=-0.2), T=T_{M}(\beta=0)$, and $T>T_{M}(\beta=0.2)$.

Figure 2.The free energy density for the isothermal solution given by (10) as a function of $\phi$ and $c$.

Figure 3. (a) The common tangent construction representing equilibrium of a stationary flat interface. (b) The parallel tangent construction predicted by this model for a non stationary planar interface. In both cases $W_{A}=W_{B}=100, \beta_{A}=-0.1$, and $\beta_{B}=0.1$.

Figure 4. The solid fraction, $S=\frac{1}{L} \int_{0}^{L} \phi d x$, versus average composition, C, obtained from from one-dimensional stationary solutions. The dashed line represents two-phase solutions. Figure 5. The solid and liquid interfacial concentrations $c_{S}$ and $c_{L}$ as a function of growth rate, $V$, for a planar constant velocity interface, for the case $M=1, W_{A}=100, W_{B}=100$, $\beta_{A}=-0.1, \beta_{B}=0.1$. The dashed curve indicates the the segregation coefficient, $k$ as a function of the growth rate.

Figure 6. The one-dimensional traveling wave solution obtained by direct numerical integration of the governing equations. The concentration profile is shown for $t=0,1,2,3$ (from left to right).

Figure 7. The evolution of the concentration profile, in response to initial conditions in which the liquid is at a spatial uniform concentration away from its equilibrium value. The concentration profile is shown at $t=0,2,4,6,8,10$.

Figure 8. The evolution of the phase field $\phi$ in time and space on the domain $[0,1] \times[0,1]$. The solid curve is the locus of points $\phi=\frac{1}{2}$ and represents the solid-liquid interface. The shaded areas represent $0<\phi<\frac{1}{4} ; \frac{1}{4}<\phi<\frac{1}{2} ; \frac{1}{2}<\phi<\frac{3}{4} ; \frac{3}{4}<\phi<1$ (in increasing order of darkness). Thus the darkest regions represent solid areas. Plots are given at times (a) $t=0$, (b) $t=0.01$, (c) $t=0.05$, (d) $t=0.1$, (e) $t=1$. 


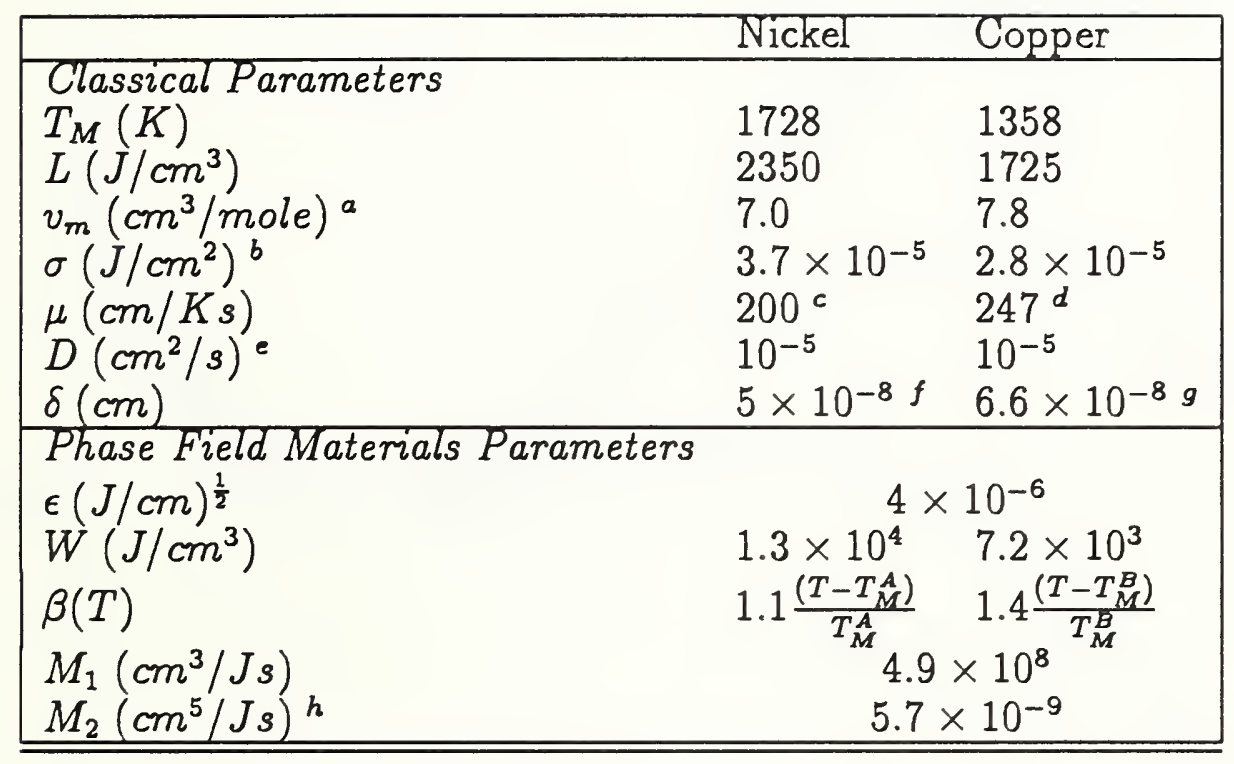

a An average value of 7.4 will be taken, since this model does not deal with volume changes.

b Estimated from $\sigma=0.7 L\left(v_{m} / N_{0}\right)^{\frac{1}{3}}$, see Coriell and Turnbull [22]; $N_{0}$ is Avagadro's Number.

c Estimated from $\mu=\frac{V_{v} L_{v m}}{R T^{2}} ; V_{s}$ is the speed of sound in liquid metal

(taken as $3 \times 10^{5} \mathrm{~cm} / \mathrm{s}$ ); Coriell and Turnbull [22].

$d$ Calculated from $\mu_{N i}$ using equation (40).

e Typical liquid diffusion coefficient.

$f$ We have taken the interface thickness to be on the order of atomic dimensions.

$g$ Calculated using equation (39).

$h$ At $T^{*}=1543 K$.

Table 1: Material and phase-field parameters for the $\mathrm{Ni}-\mathrm{Cu}$ alloy. 



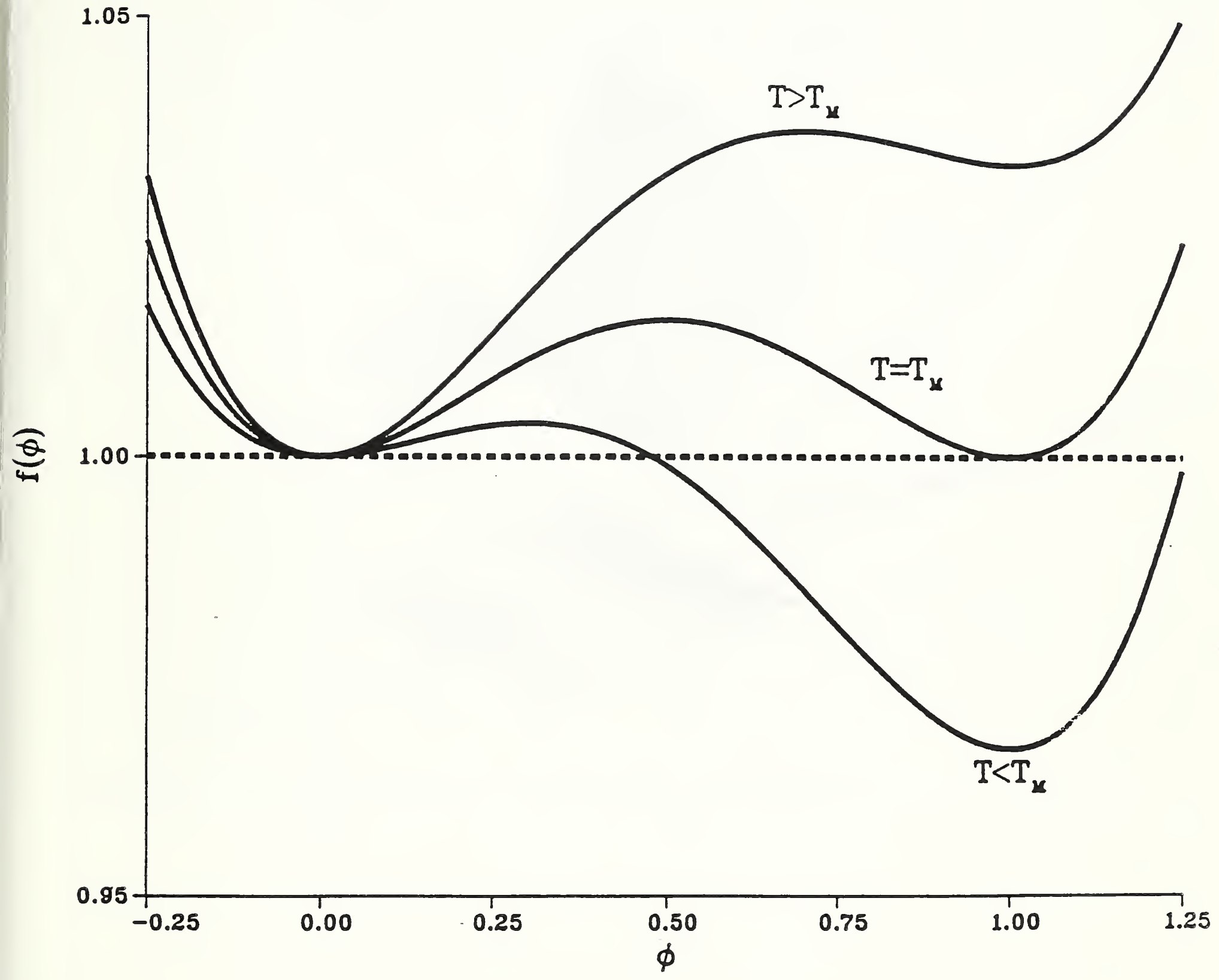

Figure 1 


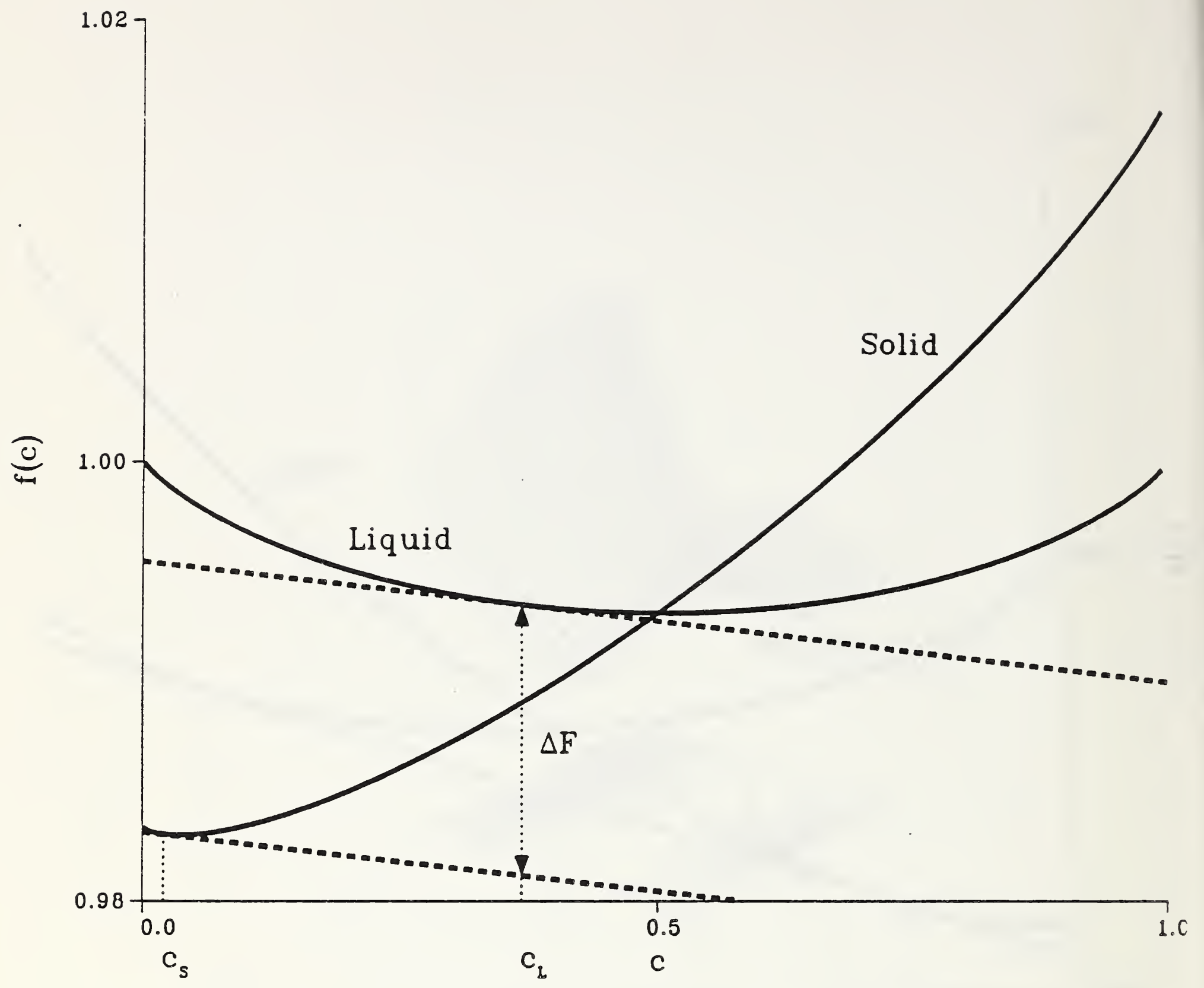

Figure 3b 


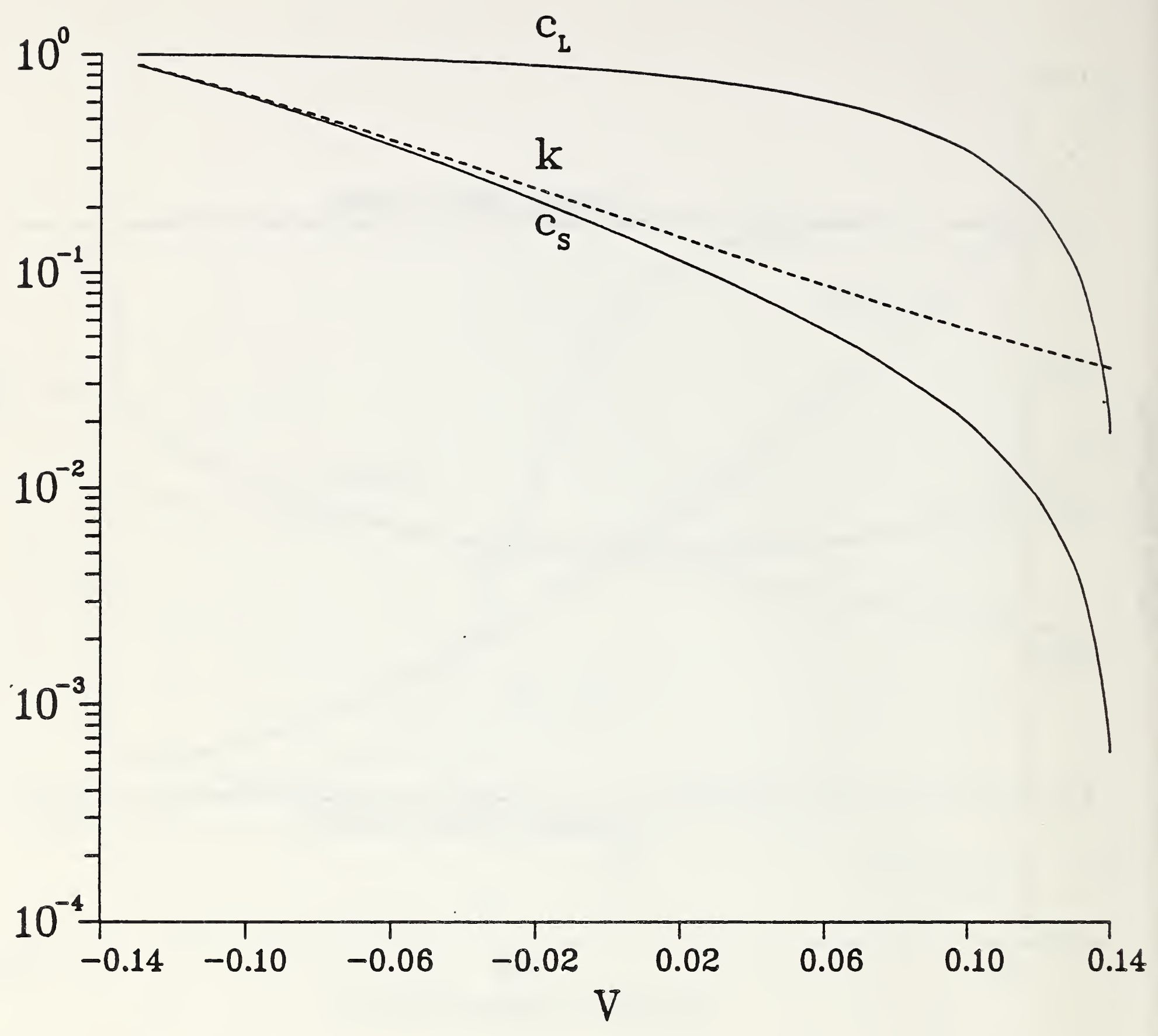

Figure 5 


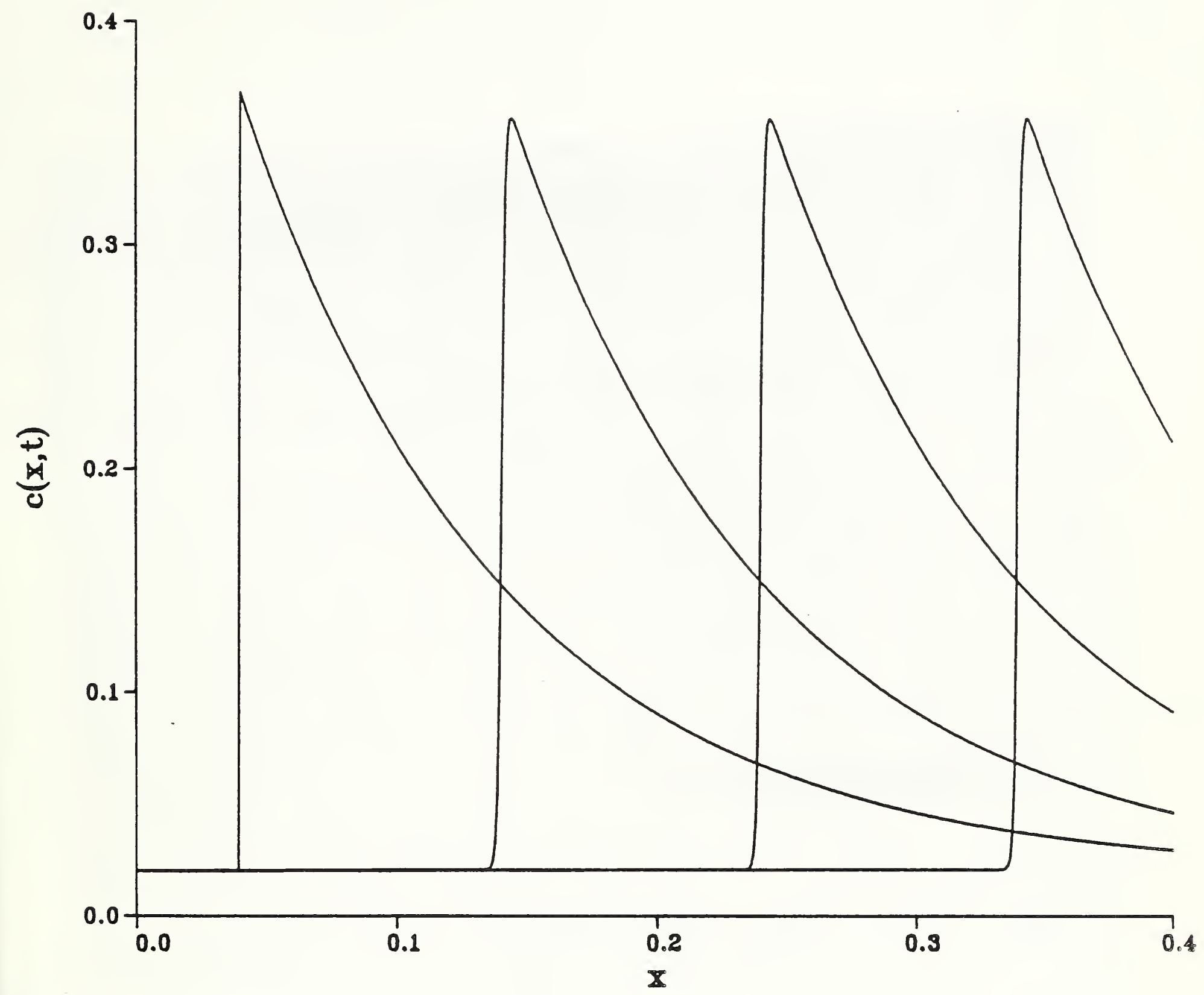

Ficure 6 


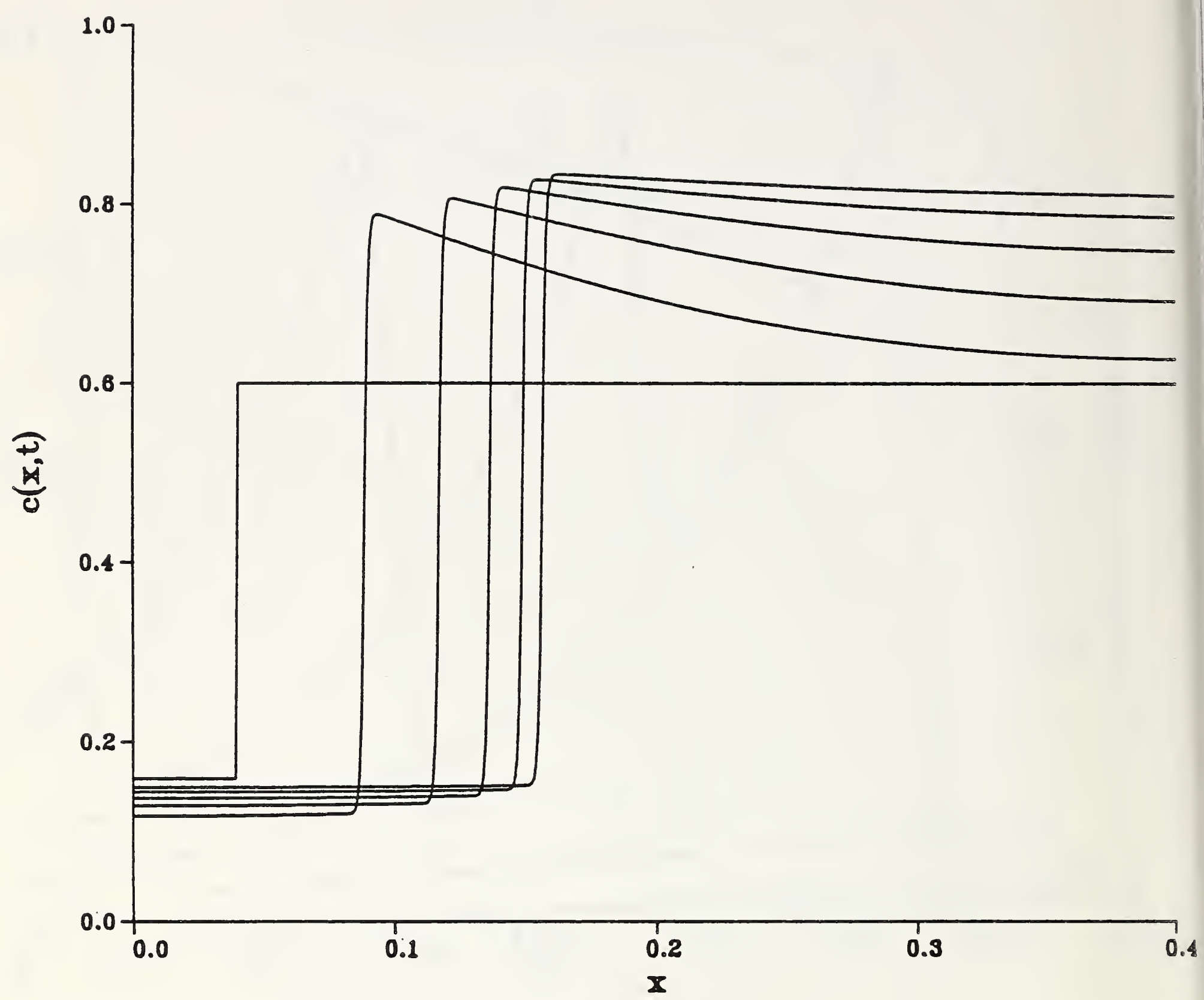

Figure ? 


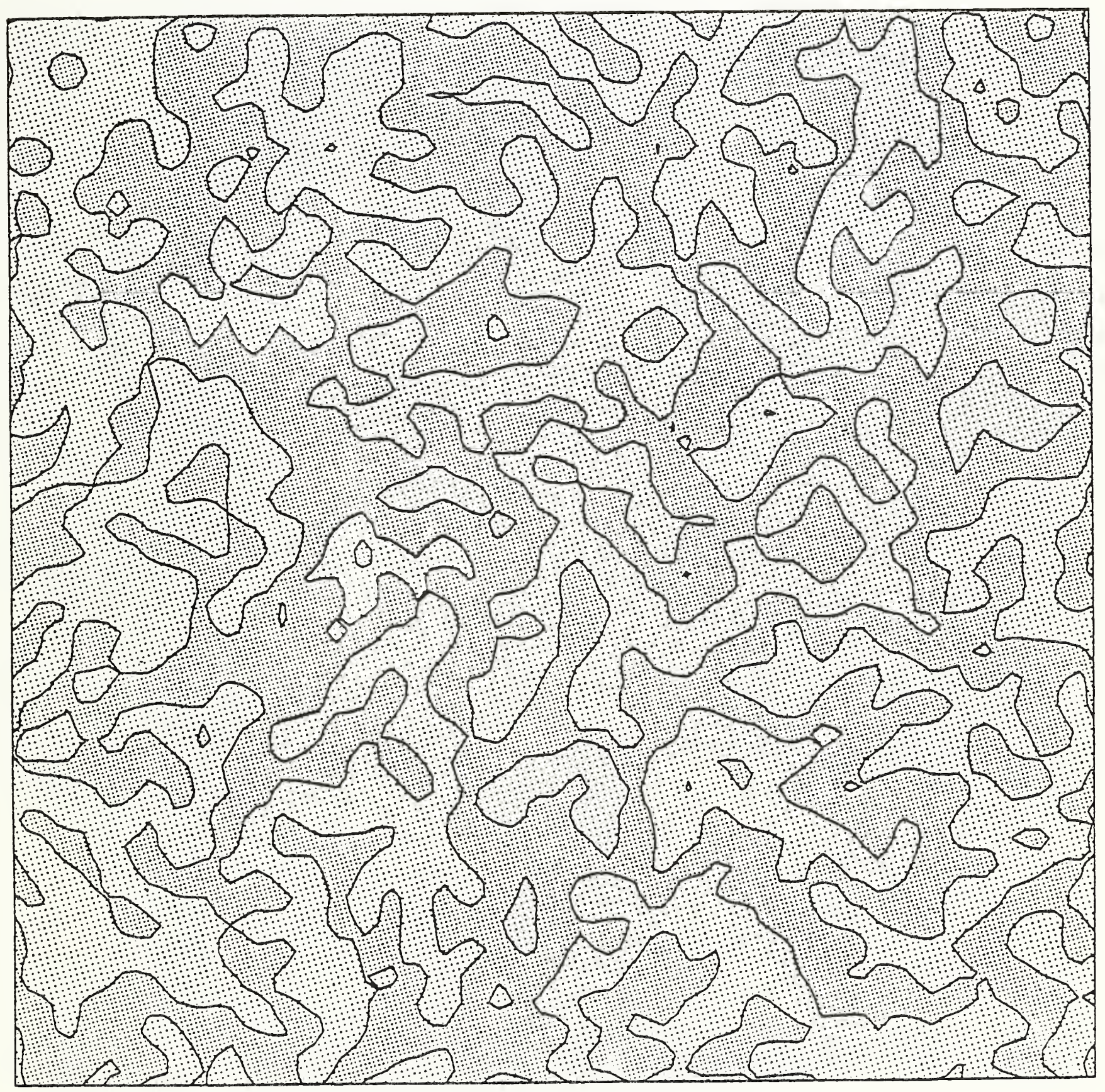




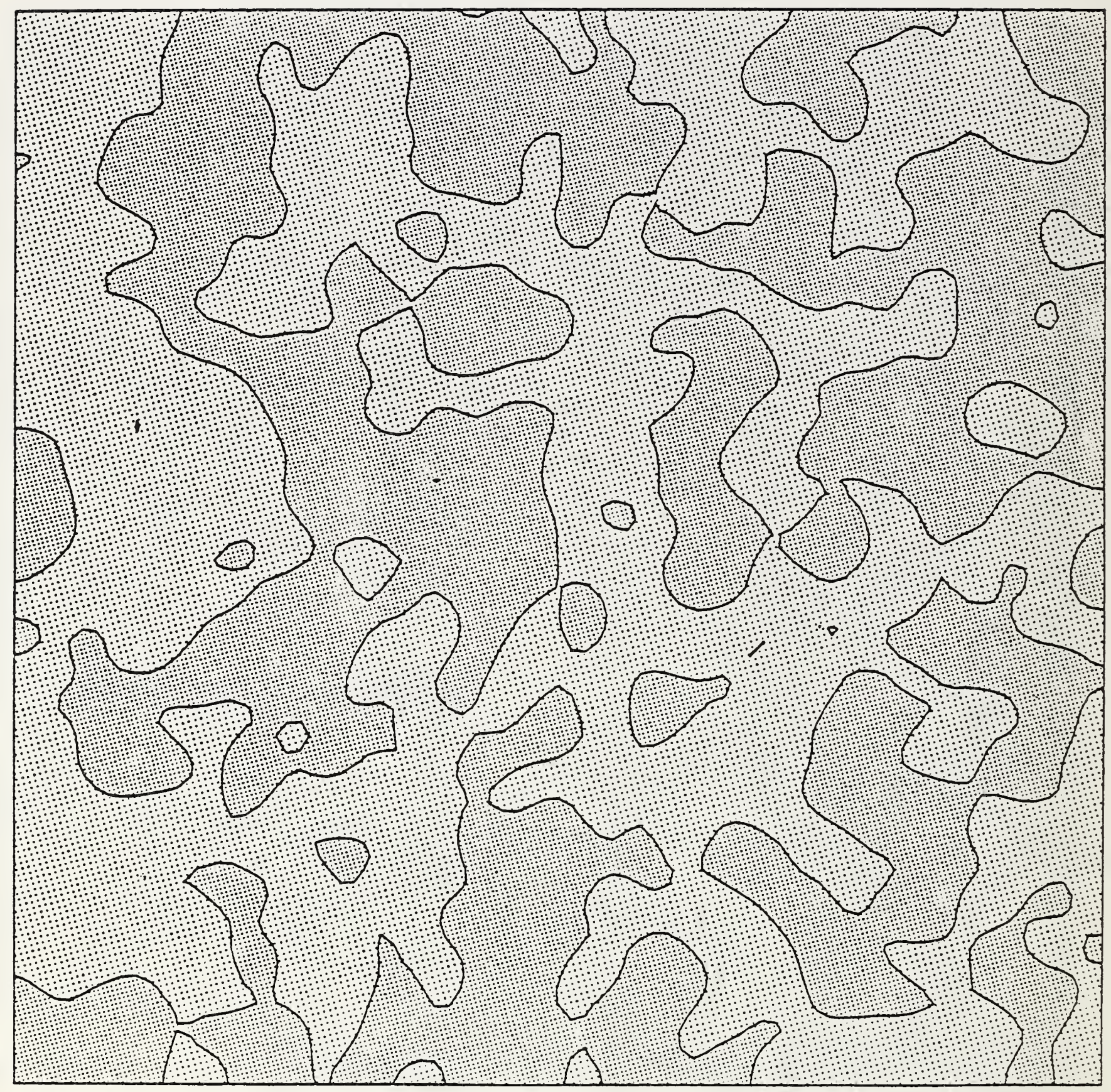




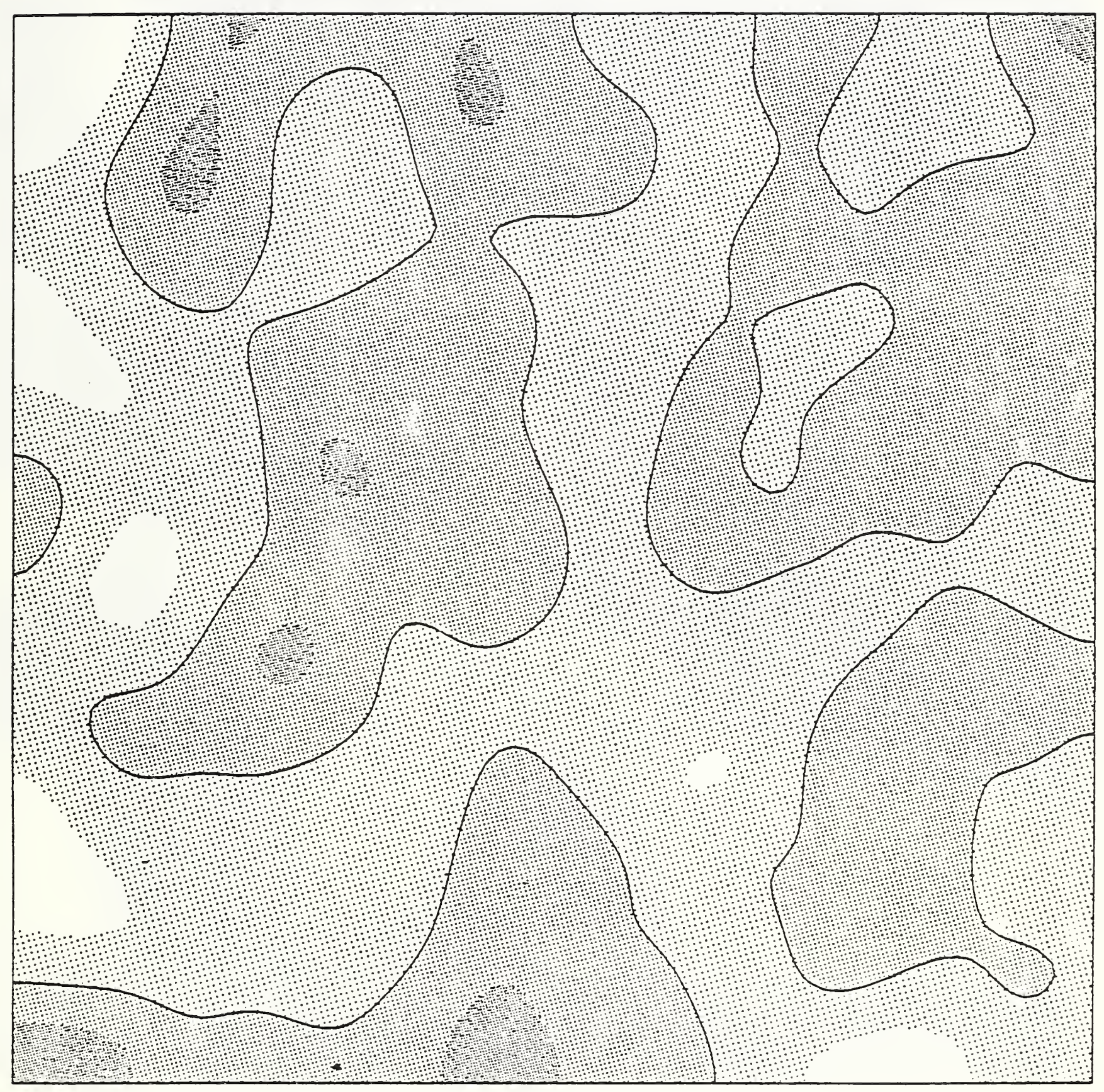




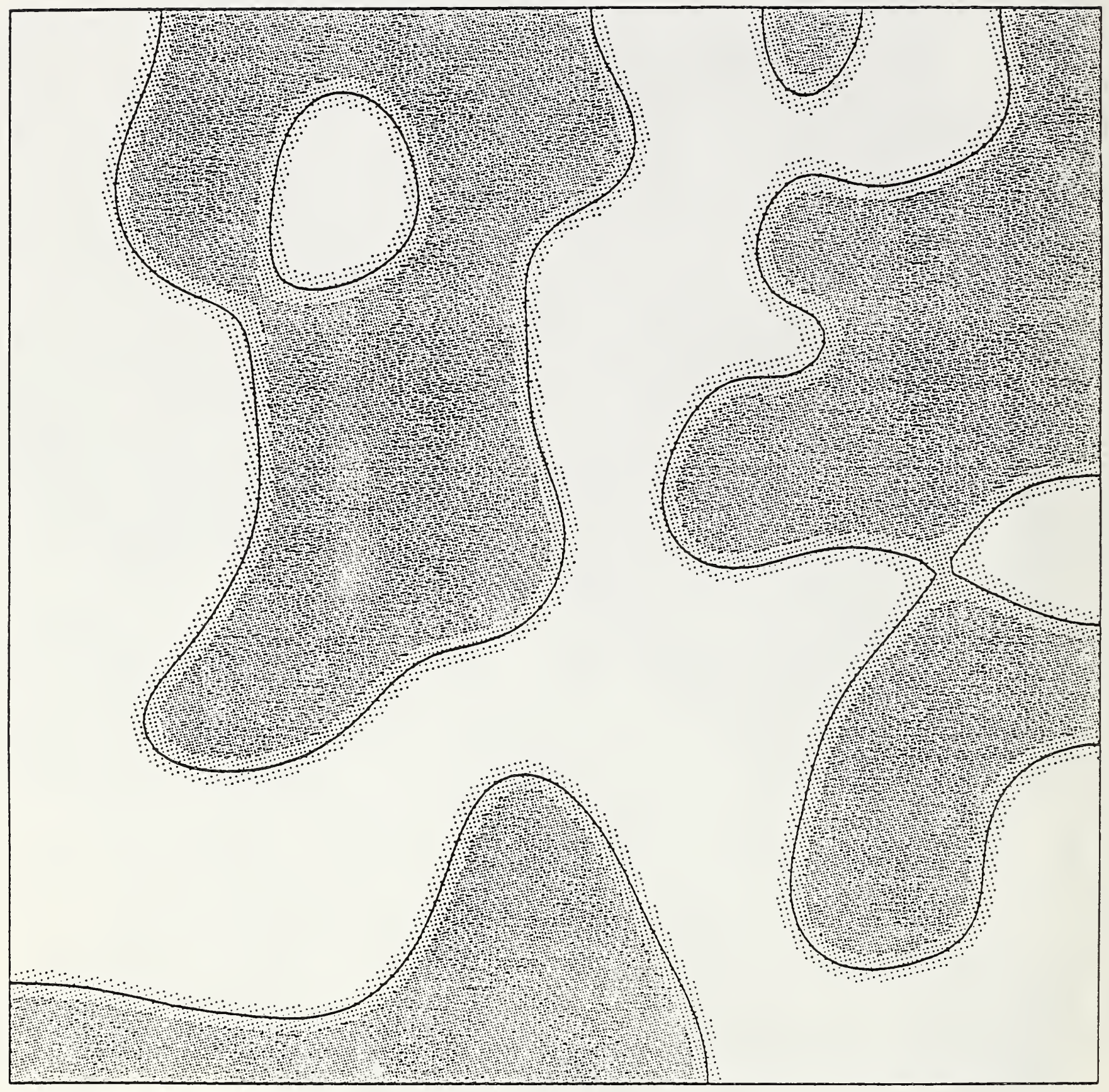




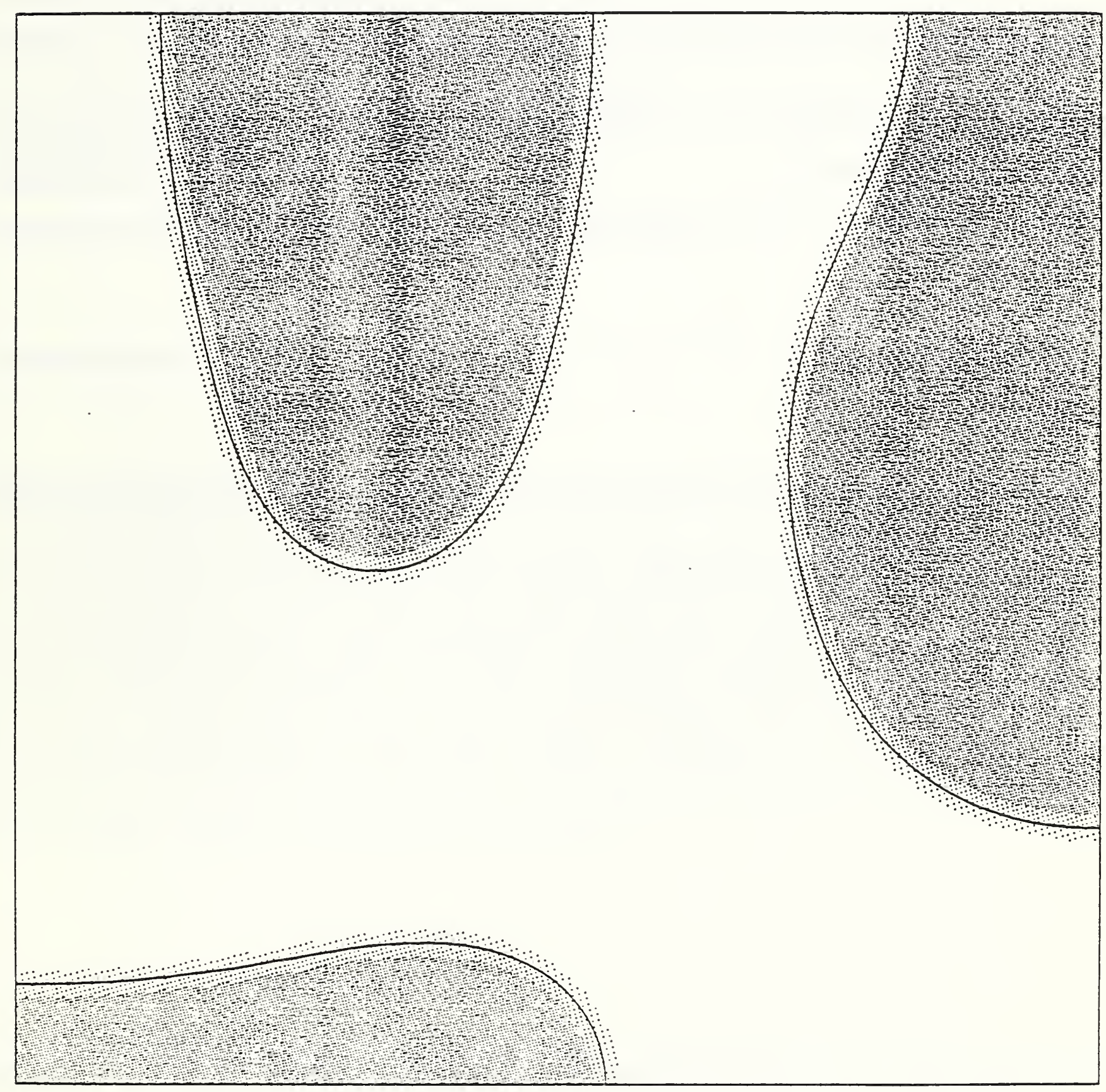

Figure $\varepsilon e$ 



\begin{tabular}{|c|c|c|}
\hline \multirow[t]{3}{*}{$\begin{array}{l}\text { NIST-114A } \\
\text { (REV. 3-90) }\end{array}$} & \multirow[t]{3}{*}{$\begin{array}{l}\text { U.S. DEPARTMENT OF COMMERCE } \\
\text { NATIONAL INSTITUTE OF STANDARDS AND TECHNOLOGY }\end{array}$} & $\begin{array}{l}\text { 1. PUBLCATION OA REPORT NUMBER } \\
\text { NISTIR } 4662\end{array}$ \\
\hline & & 2. PERFORMING ORGANIZATION REPORT NUMBEA \\
\hline & & $\begin{array}{l}\text { 3. PUBLCATION DATE } \\
\text { AUGUST } 1991\end{array}$ \\
\hline
\end{tabular}

4. TITLEAND SUBTITLE

A Phase Field Model for Isothermal Phase

Transitions in Binary Alloys

5. AUTHOR(S)

A. A. Wheeler, W. J. Boettinger, and G. B. McFadden

6. PERFORMING ORGANIZATION (IF JOINT OR OTHER THAN NIST, SEE INSTRUCTIONS)

U.S. DEPARTMENT OF COMMERCE

NATIONAL INSTITUTE OF STANDARDS AND TECHNOLOGY

GAITHERSBUAG, MD 20899

7. CONTRACT/GRANT NUMBER

8. TYPE OF REPORT AND PERIOD COVERED

9. SPONSORING ORGANIZATION HAME AND COMPLETE ADDRESS (STAEET, CITY, STATE, ZIP)

10. SUPPLEMENTARY NOTES

11. ABSTRACT (A 200-WORD OR LESS FACTUAL SUMMARY OF MOST SIGNIFICANT INFORMATION. IF DOCUMENT INCLUDES A SIGNIFICANT BIBLUOGRAPHY OR UTERATURE SURVEY, MENTION IT HERE.)

In this paper we describe a new phase field model, which models isothermal phase transitions between ideal binary alloy solution phases. Equations are developed for the temporal and spatial variation of the phase field, which describes the identity of the phase, and for the composition. We conduct an asymptotic analysis as the gradient energy coefficient of the phase field becomes small. From our analysis we show that our model recovers classical sharp interface models of this situation when the interfacial layers are thin and we show how to relate the parameters appearing in the phase field model to material and growth parameters in real systems. Further, we identify three stages of temporal evolution; the first corresponding to interfacial genesis which occurs very rapidly, the second to interfacial motion controlled by the local energy difference across the interface and diffusion; the last taking place on a long time scale in which curvature effects are important, and corresponds to ostwald ripening. We also present results of numerical calculations.

12. KEY WORDS (6 TO 12 ENTRIES; ALPHABETICAL ORDER; CAPITALLE ONLY PROPER NAMES; AND SEPARATE KEY WORDS BY SEMICOLONS) binary alloy; gradient energy; Ostwald ripening; phase field; phase transition; segregation

13. AVAILABILTY

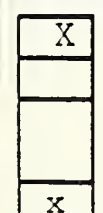

UNUMITED

FOR OFFICIAL DISTRIBUTION. DO NOT RELEASE TO NATIONAL TECHNICAL INFORMATION SERVICE (NTIS).

ORDER FROM SUPERINTENDENT OF DOCUMENTS, U.S. GOVERNMENT PRINTING OFFICE, WASHINGTON, DC 20402.

ORDER FROM NATIONAL TECHNICAL INFORMATION SEAVICE (NTIS), SPRINGFIELD, VA 22161.
14. NUMBER OF PAINTED PAGES

5]

15. PRICE

$\mathrm{A} 04$

ELECTRONIC FORM 


